\title{
CLASIFICACIÓN BIOCLIMÁTICA DE LA VERTIENTE DEL PACÍFICO MEXICANO Y SU RELACIÓN CON LA VEGETACIÓN POTENCIAL
}

\author{
Miguel Ángel Macías Rodríguez ${ }^{1,6}$, Manuel Peinado Lorca², Joaquín Giménez de \\ Azcárate ${ }^{3}$, Juan Luis Aguirre Martínez ${ }^{4}$ y José Delgadillo Rodríguez ${ }^{5}$ \\ ${ }^{1}$ Universidad de Guadalajara, Departamento de Ciencias Ambientales, \\ Carretera Guadalajara-Nogales km 15.5, 45110 Zapopan, Jalisco, México. \\ ${ }^{2}$ Universidad de Alcalá, Departamento de Ciencias de la Vida, \\ E-28871 Alcalá de Henares, España. \\ ${ }^{3}$ Universidad de Santiago de Compostela, Departamento de Botánica, \\ Escuela Politécnica Superior, E-27002 Lugo, España. \\ ${ }^{4}$ Universidad de Alcalá, Cátedra de Medio Ambiente, \\ E-28871 Alcalá de Henares, España. \\ ${ }^{5}$ Universidad Autónoma de Baja California, Facultad de Ciencias, \\ Herbario BCMEX, 22830 Ensenada, Baja California, México. \\ ${ }^{6}$ Autor para la correspondencia: mmacias@cucba.udg.mx.
}

\section{RESUMEN}

En este trabajo se abordan las relaciones entre el clima y la vegetación en el territorio de la vertiente del Pacífico Mexicano, considerada como una franja de $100 \mathrm{~km}$ de ancho que desde el litoral se extiende hacia tierra adentro y cuyos extremos norte y sur coinciden con la frontera de México con los Estados Unidos de América y con Guatemala respectivamente. Las relaciones fueron establecidas a partir del análisis bioclimático efectuado con los datos de precipitación y temperatura de 691 estaciones meteorológicas existentes en el área de estudio. La diagnosis bioclimática permitió identificar los macrobioclimas Mediterráneo y Tropical, los bioclimas Mediterráneo Pluviestacional Oceánico, Mediterráneo Xérico Oceánico, Mediterráneo Desértico Oceánico, Mediterráneo Hiperdesértico Oceánico, Tropical Pluvial, Tropical Pluviestacional, Tropical Xérico, Tropical Desértico y Tropical Hiperdesértico, y 30 isobioclimas. Los muestreos de campo se dirigieron hacia la caracterización fisionómica y florística de la vegetación natural potencial de las diferentes unidades bioclimáticas, estableciendo así los principales elementos diagnósticos (bioindicadores y fisionómico-estructurales) de la secuencia bioclimática a lo largo del gradiente latitudinal. 
Palabras clave: bioclimatología, formaciones vegetales, isobioclimas, México, vertiente del Pacífico.

\begin{abstract}
In this study we address the relationship between climate and vegetation in the territory of the Mexican Pacific rim, a strip of $100 \mathrm{~km}$ wide that extends from the coast landwards with its north and south limits coinciding with Mexico's border with the United States of America and Guatemala, respectively. Relationships were established from bioclimatic analysis performed with temperature and precipitation data from 691 weather stations existing in the study area. The bioclimatic diagnosis identified the Mediterranean and Tropical macrobioclimates, the Mediterranean Pluviseasonal Oceanic, Mediterranean Xeric Oceanic, Mediterranean Desert Oceanic, Mediterranean Hyperdesertic Oceanic, Tropical Pluvial, Tropical Pluviseasonal, Tropical Xeric, Tropical Desertic and Tropical Hiperdesertic bioclimates, and 30 isobioclimates. The field sampling was focused towards physiognomic and floristic characterization of the natural potential vegetation of the different bioclimatic units, thereby establishing the main diagnostic features (bioindicators and physiognomic-structural elements) of the bioclimatic sequence along the latitudinal gradient.
\end{abstract}

Key words: bioclimatology, isobioclimates, Mexico, plant formations, Pacific slope.

\title{
INTRODUCCIÓN
}

El clima es el principal elemento medioambiental regulador de la repartición de la vegetación mundial (Larcher, 2003). Desde los estudios geobotánicos pioneros del siglo XIX se acepta que la distribución de la cubierta vegetal está fundamentalmente determinada por factores climáticos, mientras que los edáficos juegan un papel secundario (Walter, 1985). La cuantificación de los diferentes componentes del clima es insuficiente para relacionarlo con la vegetación; por ello se han propuesto numerosos parámetros e índices que combinan temperatura, humedad, evaporación, precipitación y ritmos estacionales, entre otros.

Las limitaciones relacionadas con la distribución de las estaciones meteorológicas y con la calidad y cantidad de sus datos disponibles hacen que los análisis de sus parámetros e índices deban utilizarse conjuntamente con los de campo; de 
esta manera llegan a ser herramientas extraordinariamente útiles en el análisis de las relaciones entre clima y vegetación, y permiten trazar con exactitud las fronteras biogeográficas en territorios florísticos relativamente homogéneos (Tuhkanen, 1980), lo que en las últimas décadas impulsó el desarrollo de la Bioclimatología. Esencialmente, las clasificaciones bioclimáticas intentan clarificar las relaciones entre los valores registrados de temperatura y precipitación y la distribución geográfica de los seres vivos, en particular de las plantas y las fitocenosis (Müller, 1982; Walter, 1985).

Algunas zonas del mundo que muestran gradientes climáticos muy marcados son especialmente interesantes para determinar si la distribución de la vegetación sigue patrones que reflejen tales variaciones. En este sentido, la vertiente del Pacífico mexicano es un territorio de gran interés florístico, biogeográfico y bioclimático por los sucesivos cambios que en ella se dan. De norte a sur se produce una transición desde una zona de clima Mediterráneo, que alcanza en la Península de Baja California su límite meridional holártico, a otra donde se encuentran los desiertos más áridos de Norteamérica (Peinado et al., 1995a). Desde estos desiertos se entra en otra zona de bosques tropicales con lluvias de verano, los cuales alcanzan en la vertiente pacífica mexicana su límite septentrional de distribución en el hemisferio occidental (Brown et al., 1998; Van Devender et al., 2000; González-Elizondo et al., 2012). Hacia el extremo meridional de la franja de estudio los bosques anteriores se imbrican con los bosques de lluvia tropicales; otras formaciones típicamente tropicales, los manglares, alcanzan en las costas mexicanas de Baja California las latitudes más septentrionales dentro del reino Holártico (Peinado et al., 2008). Por citar un ejemplo, tan solo en el estado de Sonora se han reconocido cuatro regiones biogeográficas en un gradiente latitudinal norte-sur (Búrquez et al., 1999).

En contribuciones anteriores efectuadas en el oeste de Norteamérica se ha caracterizado y vinculado la distribución de la vegetación con el modelo bioclimático aquí seguido (Rivas-Martínez, 1997; Peinado et al., 1997a; Rivas-Martínez et al., 1999; Cress et al., 2009), y establecido los límites de los zonobiomas del Pacífico septentrional usando la vegetación como bioindicadora (Peinado et al., 1994a, 1997b, 2007). El presente trabajo viene a complementar el ámbito de tres publicaciones previas sobre los vínculos clima-vegetación en el oeste-noroeste de México (Peinado et al., 2010, 2011; Giménez de Azcárate et al., 2013) y tiene por objetivo presentar un análisis de la respuesta de la vegetación potencial frente a las variaciones climáticas, abordada desde un enfoque bioclimático, a lo largo de un gradiente latitudinal que cubre la vertiente del Pacífico Mexicano, utilizando para ello el sistema de clasificación global de Rivas-Martínez et al. (2011). 
Área de estudio

A lo largo de la costa del Pacífico de México, el área de estudio comprende una extensa franja de aproximadamente $8369 \mathrm{~km}$ de longitud y de $100 \mathrm{~km}$ de ancho (Fig. 1); adicionalmente se consideró el tope altitudinal de $1000 \mathrm{~m}$. Abarca desde Presa Morelos ( $\left.32^{\circ} 43^{\prime} \mathrm{N}\right)$ al norte de Baja California, hasta Ignacio López Rayón $\left(14^{\circ} 37^{\prime} \mathrm{N}\right)$ al sur de Chiapas. Longitudinalmente, la zona estudiada tiene su límite occidental cerca de Tijuana $\left(117^{\circ} 12^{\prime} \mathrm{W}\right)$, mientras que la localidad muestreada más al oriente se encuentra en Santo Domingo, Chiapas $\left(92^{\circ} 06^{\prime} \mathrm{W}\right)$.

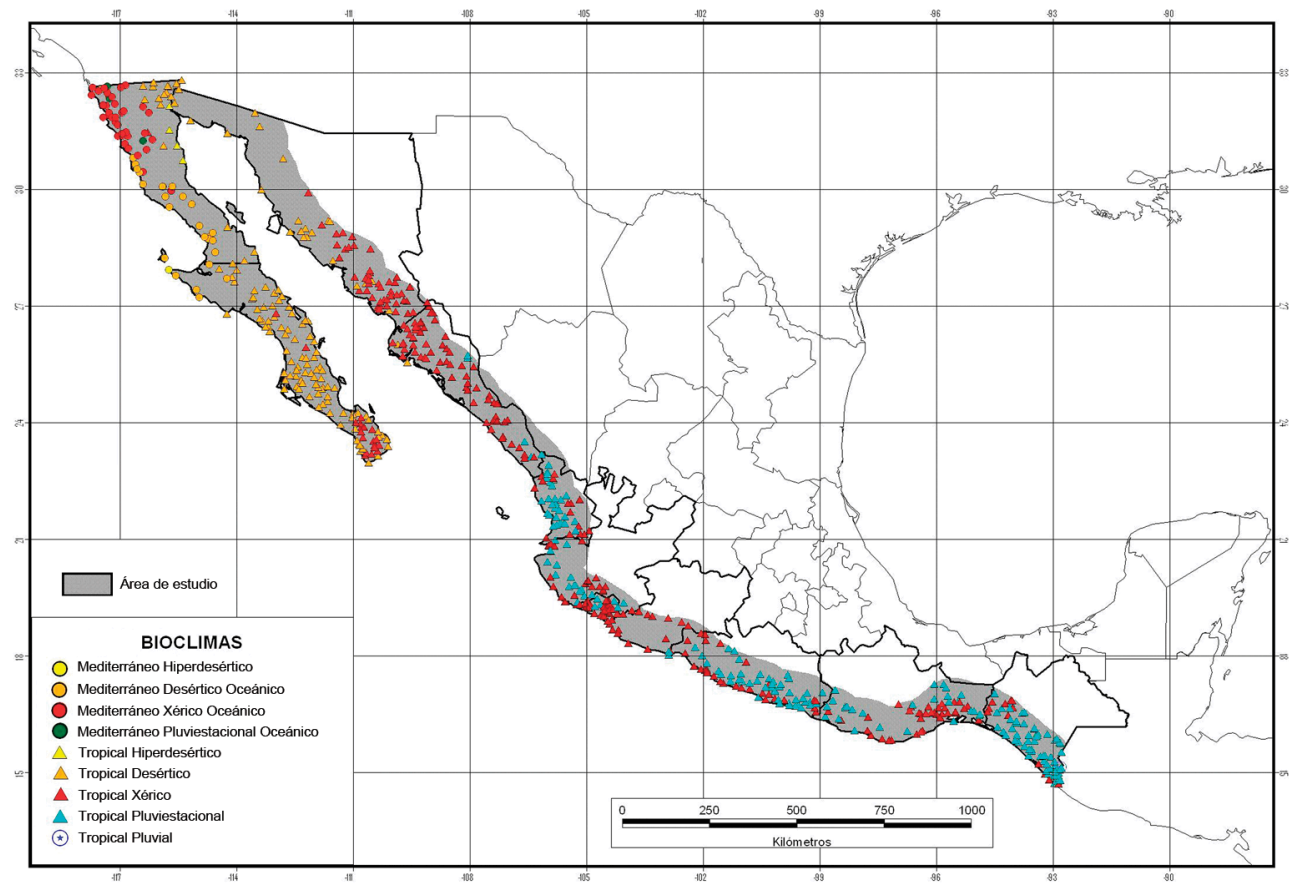

Fig. 1. Ubicación del área de estudio y la distribución de los bioclimas de las estaciones climatológicas a lo largo de la vertiente del Pacífico mexicano.

En la Península de Baja California destaca el conjunto de sierras graníticas que la recorren en toda su longitud y que condicionan el clima de ambas vertientes; así, su contacto con la costa del Pacífico es relativamente suave, lo que contrasta con la vertiente del Golfo de California, formada por un escarpe abrupto de falla, consi- 
derado el más grande de Norteamérica (Hamilton, 1971). Estas elevaciones actúan como pantallas frente a los efectos humidificadores y térmicamente refrescantes de las nieblas costeras asociadas a la corriente fría de California (Peinado et al., 2005).

En México continental, prácticamente la totalidad del área de estudio se sitúa al oeste de dos de los sistemas montañosos más grandes del país, la Sierra Madre Occidental (SMO) y la Sierra Madre del Sur (SMS). La primera transcurre paralela a la costa desde el límite septentrional del área hasta la frontera entre Nayarit y Jalisco, donde se encuentra con el Eje Volcánico Transversal (EVT). La SMS corre muy próxima a la costa, en dirección noroeste-sureste, desde el sur de Jalisco, hasta la región de los Chimalapas en el Istmo de Tehuantepec, traspasado el cual se dirige nuevamente hacia el sureste con la denominación de Sierra Madre de Chiapas (SMCh). Tales sierras protegen a la costa occidental de México de las corrientes frías que periódicamente atraviesan México de norte a sur; ello permite que las selvas tropicales alcancen su límite septentrional mucho más al norte en la vertiente del Pacífico de lo que lo hacen en la del Golfo de México (Martin y Yetman, 2000).

Aunque resulte más o menos modificado por la influencia regional o local de las cadenas montañosas y de las corrientes marinas, el clima general sigue el modelo macroclimático determinado por la circulación general de la atmósfera y, por lo tanto, está afectado por la interacción de los principales regímenes de precipitación que gobiernan la costa pacífica de México. La porción septentrional del área se sitúa dentro del cinturón subtropical anticiclónico, dominado por masas de aire descendente asociadas con células de altas presiones y cielos despejados, que provocan un pasillo conocido como la Ruta Sonorense, a través del cual masas de aire marítimo relativamente húmedo y fresco penetran durante el invierno hacia el interior árido, provocando unas ligeras precipitaciones inusuales en el resto de los desiertos norteamericanos (Bryson y Hare, 1974). De forma particular buena parte de la fachada occidental de Baja California se encuentra influenciada por la corriente fría de California y por los vientos noroccidentales, lo que provoca la aparición de una zona de clima Mediterráneo en la fachada occidental de Norteamérica (Brouillet y Whetstone, 1993).

La franja latitudinal situada entre el cinturón subtropical de altas presiones y la zona intertropical de convergencia, resulta dominada por la influencia de los alisios orientales que, procedentes del Golfo de México, recorren en verano el EVT y el Istmo de Tehuantepec. Estas corrientes mueven aire cálido y húmedo hacia el oeste hasta alcanzar la costa del Pacífico, donde se encuentran con masas convectivas que provocan el denominado "Frente Mexicano" (Mosiño y García, 1974; Douglas et al., 1993). Finalmente, el área de estudio se encuentra en el límite nororiental del complejo de tormentas tropicales y huracanes que afectan al Pacífico en otoño (Hastings y Turner, 1965). 


\section{MÉTODO}

En la selección de las estaciones meteorológicas se consideró su ubicación dentro de una banda costera de $100 \mathrm{~km}$ de ancho y por debajo de una cota de $1001 \mathrm{~m}$ de altitud; ello con el fin de reducir las modificaciones climáticas provocadas por la continentalidad y por la altitud. Además se requirió que los datos termopluviométricos tuvieran al menos 10 años de registro. Así se contabilizaron 691 estaciones cuya información fue obtenida de las siguientes fuentes: Baja California con 84 estaciones (Ruiz et al., 2006a); Baja California Sur (119) (Ruiz et al., 2006b); Chiapas (75) (Serrano et al., 2006); Colima (30) (Vizcaíno et al., 2007); Guerrero (75) (Serrano et al., 2007); Jalisco (36) (Ruiz et al., 2003); Michoacán (15) (Anguiano et al., 2005); Nayarit (49) (Anónimo, 2013); Oaxaca (66) (Serrano et al., 2005); Sinaloa (72) (Ruiz et al., 2005b) y Sonora (70) (Ruiz et al., 2005a).

A partir de dicha información se calcularon los diversos parámetros e índices bioclimáticos de la clasificación de referencia, los cuales se especifican en el Cuadro 1. La información y diagnosis bioclimática completa de cada estación meteorológica está disponible en línea (http://wwwl.inecol.edu.mx/abm/anexo). Para la obtención de los índices y de los bioclimogramas se siguió a Alcaraz (2013).

Cuadro 1. Parámetros e índices considerados en la diagnosis. Los valores de temperatura y precipitación expresados en grados Celsius y milímetros, respectivamente.

\footnotetext{
EVPT Evaporación potencial anual de Thornthwaite.

Iar Índice de aridez. Iar $=(E V P T / P)$.

Ic Índice de continentalidad/oceanidad. Equivale a la amplitud térmica anual, esto es a la diferencia entre las temperaturas medias mensuales más alta y más baja del año. Ic $=$ Tmax-Tmin.

Id Índice de diurnalidad. Equivale a la amplitud térmica diaria, esto es a la diferencia entre la temperatura media de las máximas y la temperatura media de las mínimas del mes en que estas están más alejadas (mco). Id = Tmaxmco-Tminmco.

Io Índice ombrotérmico. Io $=10(\mathrm{Pp} / \mathrm{Tp})$.

Ioe Índice de ombro-evaporación anual. Ioe $=\mathrm{P} / \mathrm{EVPT}$

$\mathbf{I o v}_{2}$ Índice ombrotérmico del mes más cálido del bimestre más cálido del trimestre estival. En territorios tropicales índice ombrotérmico de los dos meses consecutivos más secos del año.

$\mathbf{I o v}_{3} \quad$ Índice ombrotérmico del trimestre estival. En territorios tropicales índice ombrotérmico de los tres meses consecutivos más secos del año.
} 
Cuadro 1. Continuación.

\begin{tabular}{|c|c|}
\hline Iov $_{4}$ & $\begin{array}{l}\text { Índice ombrotérmico del cuatrimestre resultante de la suma del trimestre } \\
\text { estival y del mes inmediatamente anterior. En territorios tropicales índice } \\
\text { ombrotérmico resultante de la suma de los tres meses consecutivos más secos } \\
\text { del año y del mes inmediatamente anterior. }\end{array}$ \\
\hline It & Índice de termicidad. $\mathrm{It}=10(\mathrm{~T}+\mathrm{m}+\mathrm{M})$. \\
\hline M & Temperatura media de las máximas del mes más frío (mf). \\
\hline m & Temperatura media de las mínimas del mes más frío (mf). \\
\hline mco & $\begin{array}{l}\text { Mes más contrastado del año: mes con mayor diferencia entre las temperaturas } \\
\text { medias máxima y mínima. }\end{array}$ \\
\hline $\mathbf{P}$ & Precipitación anual. \\
\hline $\mathbf{P c m}_{1}$ & Precipitación del cuatrimestre más cálido del año. \\
\hline $\mathbf{P c m}_{2}$ & Precipitación del cuatrimestre anterior a $\mathrm{Pcm}_{1}$. \\
\hline $\mathbf{P c m}_{3}$ & Precipitación del cuatrimestre posterior a $\mathrm{Pcm}_{1}$. \\
\hline Pscal & Precipitación del semestre más cálido del año. \\
\hline Psfrío & Precipitación del semestre más frío del año. \\
\hline $\mathbf{T}$ & Temperatura media anual. \\
\hline Temax & Temperatura media de las máximas del mes más contrastado del año. \\
\hline Tcmin & Temperatura media de las mínimas del mes más contrastado del año. \\
\hline $\mathbf{T m}$ & Temperatura media mensual. \\
\hline Tmax & Temperatura media del mes más cálido del año. \\
\hline Tmin & Temperatura media del mes más frío del año. \\
\hline Tn & $\begin{array}{l}\text { Temperatura negativa anual: Suma en décimas de grados de las temperaturas } \\
\text { medias mensuales de los meses cuya temperatura media es inferior a } 0^{\circ} \mathrm{C} \text {. }\end{array}$ \\
\hline $\mathbf{T p}$ & $\begin{array}{l}\text { Temperatura anual positiva. Suma en décimas de grados de las temperaturas } \\
\text { mensuales medias de los meses cuya temperatura media es superior a } 0^{\circ} \mathrm{C} \text {. }\end{array}$ \\
\hline
\end{tabular}

Los bioclimogramas constituyen una manera gráfica de representar conjuntamente en un sistema de coordenadas cartesianas provisto de una doble escala de ordenadas $(\mathrm{P}=2 \mathrm{~T})$, la temperatura y la pluviosidad media mensuales a lo largo de 12 meses del año, por lo que su finalidad es la representación gráfica de las características macroclimáticas de una estación meteorológica (Del Río-González, 2005).

El sistema de clasificación bioclimática global está basado en cuatro unidades tipológicas jerarquizadas: Macrobioclima, Bioclima, Termotipo y Ombrotipo. Los bioclimas representan la unidad básica de referencia tipológica, debajo de la de mayor rango que es el Macrobioclima, y se delimitan en función de los índices 
ombrotérmicos. La clave para la determinación de los bioclimas mediterráneos y tropicales, así como los parámetros, índices y definiciones de la clasificación pueden consultarse en la propuesta de clasificación bioclimática global de Rivas-Martínez et al. (2011). A este respecto se recogen en los Cuadros 2 y 3 los termotipos y ombrotipos reconocidos en la zona de estudio. Finalmente, la definición bioclimática de cualquier territorio se hace en función de su isobioclima, un modelo bioclimático integrador formado por un bioclima, un termotipo y un ombrotipo. Los isobioclimas de la zona de estudio se plasman en el Cuadro 4.

Para el establecimiento de la reciprocidad entre el clima y la vegetación se ha seguido la propuesta de Beard (1973) relacionada con la estructura y la fisionomía de esta última, a la que se añadió la diagnosis florística de las especies dominan-

Cuadro 2. Termotipos y horizontes reconocidos en la zona de estudio con sus correspondientes valores de It (Índice de termicidad).

\begin{tabular}{ll|ll}
\hline Termotipo y horizonte & $I t$ & Termotipo y horizonte & $I t$ \\
\hline Infratropical inferior & $>800$ & Inframediterráneo inferior & $515-580$ \\
Infratropical superior & $710-800$ & Inframediterráneo superior & $450-515$ \\
Termotropical inferior & $600-710$ & Termomediterráneo inferior & $400-450$ \\
Termotropical superior & $490-600$ & Termomediterráneo superior & $350-400$ \\
Mesotropical inferior & $405-490$ & Mesomediterráneo inferior & $285-350$ \\
Mesotropical superior & $320-405$ & & \\
\hline
\end{tabular}

Cuadro 3. Ombrotipos y horizontes reconocidos en la zona de estudio con sus correspondientes valores de Io (Índice ombrotérmico anual).

\begin{tabular}{ll|ll}
\hline Ombrotipos & $I o$ & Ombrotipos & $I o$ \\
\hline Ultrahiperárido inferior & $0.0-0.1$ & Seco inferior & $2.0-2.8$ \\
Ultrahiperárido superior & $0.1-0.2$ & Seco superior & $2.8-3.6$ \\
Hiperárido inferior & $0.2-0.3$ & Subhúmedo inferior & $3.6-4.8$ \\
Hiperárido superior & $0.3-0.4$ & Subhúmedo superior & $4.8-6.0$ \\
Árido inferior & $0.4-0.7$ & Húmedo inferior & $6.0-9.0$ \\
Árido superior & $0.7-1.0$ & Húmedo superior & $9.0-12.0$ \\
Semiárido inferior & $1.0-1.5$ & Hiperhúmedo inferior & $12.0-18.0$ \\
Semiárido superior & $1.5-2.0$ & Hiperhúmedo superior & $18.0-24.0$ \\
\hline
\end{tabular}


tes. En la selección de las parcelas se buscó la homogeneidad florística (Westhoff y van der Maarel, 1973) y se consideró el concepto de vegetación potencial natural (Tüxen, 1956; Rzedowski, 1978; Rivas-Martínez, 1997; Géhu, 2006), por ser ésta la que se ajusta de forma más precisa en el establecimiento de dicha reciprocidad. La aplicación de este enfoque facilita la interpretación de los resultados especialmente en zonas cuya flora no se conoce perfectamente o cuando la información relativa a la misma está tan dispersa que la hace inaplicable en estudios que exceden el ámbito local. Así, tras la caracterización bioclimática de las estaciones y tras el trabajo de campo, se procedió a asignar a cada estación su correspondiente vegetación potencial; de forma complementaria, en algunos casos se consideró información procedente de mapas de vegetación (Leopold, 1950; Rzedowski, op. cit; Anónimo, 1995a, b, 1997, 2001, 2002).

Entre 2007 y 2012 se visitó $90 \%$ de las estaciones meteorológicas antes y después del período de lluvias con el objetivo de identificar las variaciones fisionómicas más significativas de la vegetación. Cuando la vegetación estaba bien conservada se analizaron parcelas vecinas a cada estación. En caso contrario, los datos se tomaron en localidades próximas que fueron escogidas tras examinar imágenes a través de la plataforma Google Earth (2013); ello con el fin de dirigir la prospección y así poder verificar la existencia de vegetación primaria en buen estado de conservación. En los muestreos se caracterizaron los estratos presentes, se inventariaron las especies diagnósticas, y se determinó su biotipo y características ecofisionómicas (Box, 1981); de forma complementaria se obtuvieron datos de altitud, pendiente, exposición del terreno, tipo de suelo y sustrato geológico. Los datos edáficos y geológicos fueron tomados de Anónimo (1995a, b, 1997, 2001, 2002). Las especies vegetales fueron determinadas usando algunas floras regionales: Gentry (1942), Shreve y Wiggins (1964), Wiggins (1980), Felger (2000), Pennington y Sarukhán (2005), entre otras. La nomenclatura sigue a Tropicos (2013).

\section{RESULTADOS}

Clasificación bioclimática

Las 691 estaciones meteorológicas analizadas se incluyen en dos macrobioclimas, Mediterráneo y Tropical, y en nueve bioclimas, Mediterráneo Oceánico (M. O.) Hiperdesértico, M. O. Desértico, M. O. Xérico, M. O. Pluviestacional, Tropical (T.) Hiperdesértico, T. Desértico, T. Xérico, T. Pluviestacional y T. Pluvial. Los termotipos representados son seis, Inframediterráneo, Termomediterráneo, Meso- 
mediterráneo, Infratropical, Termotropical y Mesotropical, y ocho los ombrotipos: Ultrahiperárido, Hiperárido, Árido, Semiárido, Seco, Subhúmedo, Húmedo e Hiperhúmedo. Las combinaciones encontradas de bioclimas, termotipos y ombrotipos arrojan un total de 30 isobioclimas. En la Figura 1 se refleja la distribución de las estaciones de acuerdo con su correspondiente bioclima; como complemento de lo anterior, en la Figura 2 se presentan cuatro bioclimogramas representativos de los dos macrobioclimas presentes.

El Cuadro 4 permite comparar y discriminar los parámetros clave de los 30 isobioclimas presentes en el área de estudio. En línea (http://wwwl.inecol.edu.mx/ abm/anexo), se ofrecen datos florísticos de los tipos de vegetación potencial reconocidos, junto con referencias bibliográficas, lo que permite comparar la clasificación bioclimática con las denominaciones más difundidas de los tipos de vegetación mexicanos. En la misma dirección electrónica, se incluyen todos los bioclimogramas de cada una de las estaciones meteorológicas registradas en cada uno de los estados.

El Macrobioclima Mediterráneo se distribuye grosso modo en el cuadrante noroccidental de la Península de Baja California con 65 estaciones registradas; el resto de las estaciones de la Península (138) pertenecen al Tropical. Los bioclimas e isobioclimas reconocidos, así como el número de estaciones meteorológicas registradas para cada situación, y el tipo de vegetación potencial asociado a cada caso se refleja en el Cuadro 5. Dentro del bioclima Mediterráneo Hiperdesértico Oceánico (MEHO), se presenta el isobioclima MEHO Inframediterráneo Ultrahiperárido, que corresponde con la formación vegetal denominada Vegetación de Suelos Alcalinos (ALCA), cuya distribución se circunscribe a los territorios más occidentales del Desierto del Vizcaíno, con una única estación representada (Punta Eugenia).

El bioclima Mediterráneo Desértico Oceánico (MEDO) se presenta en la porción meridional del territorio mediterráneo peninsular, aproximadamente por debajo del paralelo $31^{\circ} \mathrm{N}$; se reconocen los termotipos Inframediterráneo y Termomediterráneo, cada uno con los ombrotipos Hiperárido y Árido. El isobioclima MEDO Inframediterráneo Hiperárido está representado por las formaciones ALCA y Desierto Sarcocaulescente (DESA), distribuidos en la Isla Cedros y al sur del Desierto del Vizcaíno. En el isobioclima MEDO Termomediterráneo Hiperárido se desarrolla también el DESA cuya estación de referencia es Ejido José María Morelos, al norte del Desierto de Vizcaíno. Para los pisos Inframediterráneo y Termomediterráneo con ombrotipo Árido se desarrollan las formaciones DESA y Matorral Costero (MACO) cubriendo la franja suroeste de la vertiente pacifica mediterránea de Baja California, desde la estación Colonia Guerrero hasta la esta- 
Macías Rodríguez et al.: Clasificación bioclimática de la vertiente del Pacífico mexicano
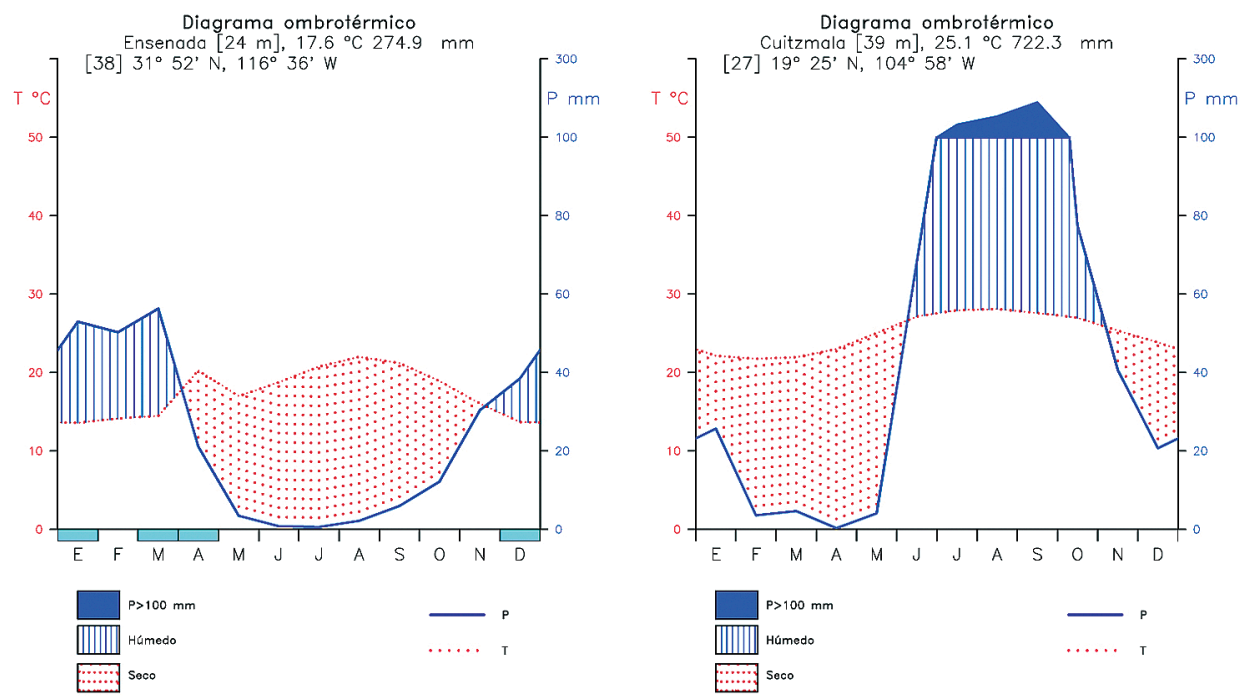

Mediterráneo Xérico Oceánico

Termomediterráneo Semiárido

\section{Tropical Xérico \\ Termotropical Seco}

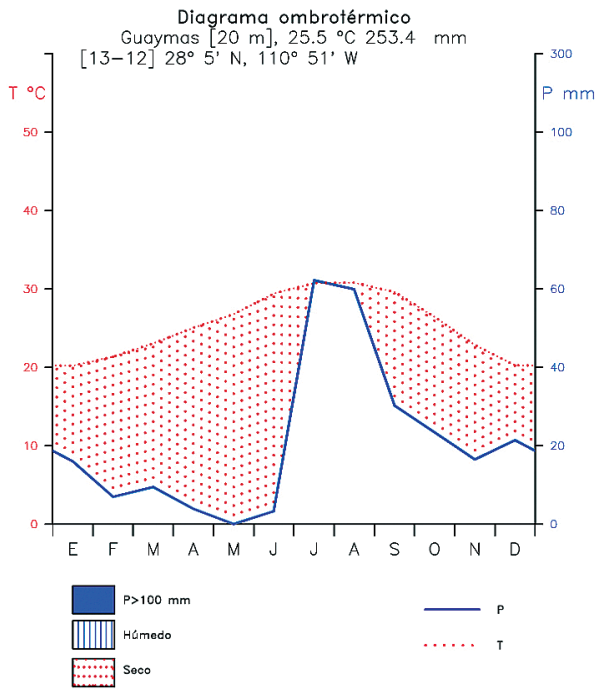

Tropical Desértico Termotropical Árido

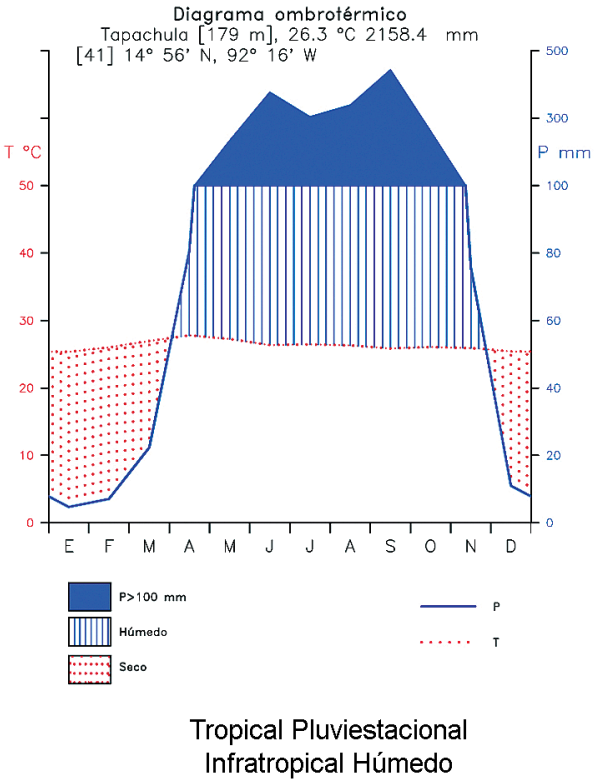

Fig. 2. Ejemplos de bioclimogramas de cuatro estaciones climatológicas correspondientes a los dos Macrobioclimas encontrados en la zona de estudio. $\mathrm{P}=$ Precipitación; $\mathrm{T}=$ Temperatura y $\mathrm{H}=$ Helada. 
Cuadro 4. Clave de identificación para los isobioclimas existentes en el área de estudio. Abreviaturas como en el Cuadro 1. Los números entre paréntesis después de cada isobioclima indican el número de estaciones que lo presentan.

\begin{tabular}{|c|c|}
\hline 1 Latitud $>26^{\circ} 30^{\prime} \mathrm{N}$ & 2 \\
\hline Latitud $\leq 26^{\circ} 30^{\prime} \mathrm{N}$ & 7 \\
\hline $\begin{array}{l}2 \mathrm{Al} \text { menos deben cumplirse } \\
\text { dos de las siguientes tres } \\
\text { condiciones: } \mathrm{T} \geq 25^{\circ} ; \mathrm{m} \geq 10^{\circ} ; \\
\text { It } \geq 580 \text {. }\end{array}$ & 7 \\
\hline No cumple las condiciones & 3 \\
\hline $\begin{array}{l}3 \mathrm{Al} \text { menos deben cumplirse } \\
\text { dos de las siguientes tres } \\
\text { condiciones: } \mathrm{T}>21^{\circ} ; \mathrm{M}>18^{\circ} \\
\text { It } \geq 470 \text {. }\end{array}$ & 4 \\
\hline No cumple las condiciones & 6 \\
\hline 4 Pscal > Psfrío & 7 \\
\hline Pscal $<$ Psfrío & 5 \\
\hline $\begin{array}{r}5 \operatorname{Ios}_{2}>2 \\
\operatorname{Ios}_{2} \leq 2\end{array}$ & 78 \\
\hline $\begin{array}{l}6 \mathrm{Pcm}_{2}<\mathrm{Pcm}_{1}>\mathrm{Pcm}_{3} \\
\text { No cumple las condiciones }\end{array}$ & $\begin{array}{l}7 \\
26 \\
\end{array}$ \\
\hline $\begin{array}{r}7 \text { Io }>3.6 \\
\text { Io } \leq 3.6\end{array}$ & 8 \\
\hline $\begin{array}{r}8 \operatorname{Ios}_{2}>2.5 \\
\operatorname{Ios}_{2} \leq 2.5 \\
\end{array}$ & \begin{tabular}{|l} 
Tropical Pluvial Infratropical Hiperhúmedo (1) \\
$\mathbf{1 0}$
\end{tabular} \\
\hline 9 Io $>1$ & 16 \\
\hline Io $\leq 1$ & 21 \\
\hline 10 It $>710$ & 11 \\
\hline It $\leq 710$ & 13 \\
\hline $\begin{array}{r}1 \text { Io }>12 \\
\text { Io }<12 \\
\end{array}$ & $\begin{array}{l}\text { Tropical Pluviestacional Infratropical Hiperhúmedo (5) } \\
\mathbf{1 2}\end{array}$ \\
\hline 12 Io $>6$ & Tropical Pluviestacional Infratropical Húmedo (19) \\
\hline Io $\leq 6$ & Tropical Pluviestacional Infratropical Subhúmedo (71) \\
\hline $\begin{array}{r}13 \text { It } \leq 490 \\
\text { It }>490\end{array}$ & $\begin{array}{l}\text { Tropical Pluviestacional Mesotropical Subhúmedo (4) } \\
\mathbf{1 4}\end{array}$ \\
\hline $\begin{array}{r}14 \text { Io }>12 \\
\text { Io } \leq 12 \\
\end{array}$ & $\begin{array}{l}\text { Tropical Pluviestacional Termotropical Hiperhúmedo (3 } \\
\mathbf{1 5}\end{array}$ \\
\hline $\begin{array}{r}15 \text { Io }>6 \\
\text { Io } \leq 6\end{array}$ & $\begin{array}{l}\text { Tropical Pluviestacional Termotropical Húmedo (25) } \\
\text { Tropical Pluviestacional Termotropical Subhúmedo (66) }\end{array}$ \\
\hline
\end{tabular}


Macías Rodríguez et al.: Clasificación bioclimática de la vertiente del Pacífico mexicano

Cuadro 4. Continuación.

\begin{tabular}{|c|c|}
\hline $\begin{array}{r}16 \text { It }>710 \\
\text { It } \leq 710\end{array}$ & $\begin{array}{l}17 \\
18\end{array}$ \\
\hline 17 Io $>2$ & Tropical Xérico Infratropical Seco (95) \\
\hline Io $\leq 2$ & Tropical Xérico Infratropical Semiárido (9) \\
\hline 18 It $>490$ & 19 \\
\hline It $\leq 490$ & 20 \\
\hline 19 Io $>2$ & Tropical Xérico Termotropical Seco (97) \\
\hline Io $\leq 2$ & Tropical Xérico Termotropical Semiárido (82) \\
\hline 20 Io $>2$ & Tropical Xérico Mesotropical Seco (3) \\
\hline Io $\leq 2$ & Tropical Xérico Mesotropical Semiárido (4) \\
\hline 21 Io $<0.2$ & 22 \\
\hline Io $>0.2$ & 23 \\
\hline 22 It $>490$ & $\begin{array}{l}\text { Tropical Hiperdesértico Termotropical Ultrahiperárido } \\
\text { (2) }\end{array}$ \\
\hline It $>490$ & Tropical Hiperdesértico Mesotropical Ultrahiperárido (2) \\
\hline 23 It $>490$ & 24 \\
\hline It $\leq 490$ & 25 \\
\hline 24 Io $>0.4$ & Tropical Desértico Termotropical Árido (91) \\
\hline Io $\leq 0.4$ & Tropical Desértico Termotropical Hiperárido (27) \\
\hline 25 Io $>0.4$ & Tropical Desértico Mesotropical Árido (9) \\
\hline Io $\leq 0.4$ & Tropical Desértico Mesotropical Hiperárido (11) \\
\hline 26 Io $>2$ & 27 \\
\hline Io $\leq 2$ & 28 \\
\hline 27 It $>350$ & $\begin{array}{l}\text { Mediterráneo Pluviestacional Oceánico } \\
\text { Termomediterráneo Seco (2) }\end{array}$ \\
\hline It $\leq 350$ & $\begin{array}{l}\text { Mediterráneo Pluviestacional Oceánico } \\
\text { Mesomediterráneo Seco (1) }\end{array}$ \\
\hline 28 Io $>1$ & 29 \\
\hline Io $<1$ & 31 \\
\hline 29 It $>450$ & $\begin{array}{l}\text { Mediterráneo Xérico Oceánico Inframediterráneo } \\
\text { Semiárido (4) }\end{array}$ \\
\hline It $\leq 450$ & 30 \\
\hline 30 It $>350$ & $\begin{array}{l}\text { Mediterráneo Xérico Oceánico Termomediterráneo } \\
\text { Semiárido (28) }\end{array}$ \\
\hline It $\leq 350$ & $\begin{array}{l}\text { Mediterráneo Xérico Oceánico Mesomediterráneo } \\
\text { Semiárido (5) }\end{array}$ \\
\hline 31 Io $<0.2$ & $\begin{array}{l}\text { Mediterráneo Hiperdesértico Oceánico } \\
\text { Inframediterráneo Ultrahiperárido (1) }\end{array}$ \\
\hline Io $\geq 0.2$ & 32 \\
\hline
\end{tabular}


Cuadro 4. Continuación.

\begin{tabular}{c|l}
\hline $\mathbf{3 2}$ It $>450$ & $\mathbf{3 2}$ \\
It $\leq 450$ & $\mathbf{3 4}$ \\
\hline $\mathbf{3 3}$ Io $>0.4$ & Mediterráneo Desértico Oceánico Inframediterráneo \\
& Árido (9) \\
Io $\leq 0.4$ & Mediterráneo Desértico Oceánico Inframediterráneo \\
& Hiperárido (5) \\
\hline $\mathbf{3 4}$ Io $>0.4$ & Mediterráneo Desértico Oceánico Termomediterráneo \\
& Árido (9) \\
Io $\leq 0.4$ & Mediterráneo Desértico OceánicoTermomediterráneo \\
& Hiperárido (1) \\
\hline
\end{tabular}

ción Rancho Alegre. Los bioclimas MEDO Y MEHO dominan al sur del paralelo $30^{\circ} \mathrm{N}$, a través de las llanuras de El Vizcaíno y del desierto Central, donde se dejan sentir las influencias marítimas ya muy atenuadas de los vientos del noroeste y de los alisios orientales. Estos territorios constituyen la zona de transición hacia el Macrobioclima Tropical.

El bioclima Mediterráneo Xérico Oceánico (MEXO) se distribuye en la porción noroccidental de la Península entre los paralelos $31^{\circ}$ y $33^{\circ} \mathrm{N}$; se reconocen los pisos Inframediterráneo, Termomediterráneo y Mesomediterráneo, todos bajo ombrotipo Semiárido; el primero se corresponde con las formaciones dominadas por MACO; en el Termomediterráneo se presenta la formación de Chaparral (CHAPA), mientras que en el Mesomediterráneo se corresponde con la del Pinar Mediterráneo (PIME).

En el bioclima Mediterráneo Pluviestacional Oceánico (MEPO) se reconocen los pisos Termomediterráneo y Mesomediterráneo con ombrotipo Seco y aparecen las comunidades CHAPA, PIME y Encinar Perennifolio Mediterráneo (ENPE), las cuales son más templadas y húmedas que las anteriormente comentadas. Este bioclima está pobremente representado con tan solo tres estaciones meteorológicas, ya que su óptimo se ubica en cotas más elevadas que las aquí consideradas. Las comunidades incluidas en CHAPA y PIME también llegan a desarrollarse bajo los isobioclimas MEXO Termomediterráneo Semiárido y Mesomediterráneo Semiárido que, al igual que los anteriores, se presentan en el piedemonte y en las laderas occidentales de las Sierras Juárez y San Pedro Mártir. Para descripciones más detalladas acerca de la vegetación del macrobioclima 
Macías Rodríguez et al.: Clasificación bioclimática de la vertiente del Pacífico mexicano

Mediterráneo en Baja California cabe remitirse a Peinado et al. (1990, 1994a, b, c, 1995d, 1997a, 2008).

En el Cuadro 5, necesariamente resumido, se presenta los resultados de la clasificación utilizada; téngase en cuenta que cuando en algunos de los isobioclimas mediterráneos aparezcan tipos fisionómicos dominados o codominados por Larrea tridentata, su tropicalidad florística es acusada.

Cuadro 5. Macrobioclimas (MB), Bioclimas, Isobioclimas, Número de estaciones (NE) y su relación con su vegetación potencial presentes dentro del área de estudio. ALCA: Vegetación de suelos alcalinos; BTCA: Bosque tropical caducifolio; BTPE: Bosque Tropical Perennifolio; BTSP: Bosque Tropical Semiperennifolio; CHAP: Chaparral; DEAF: Desierto Arbosufrutescente; DECR: Desierto Crasicaulescente; DEMI: Desierto Microfilo; DESA: Desierto Sarcocaulescente; ENCA: Encinar Caducifolio Tropical; ENPE: Encinar Perennifolio Mediterráneo; MACO: Matorral Costero; MAES/BOES: Matorral y Bosque Espinosos; MANG: Manglar; MESQ: Mezquital; PALM: Palmar; PIME: Pinar Mediterráneo; PITR: Pinar Tropical. El paréntesis indica el número de estaciones representativas de cada caso.

\begin{tabular}{|c|c|c|c|c|}
\hline MB & BIOCLIMA & ISOBIOCLIMA & $\mathrm{NE}$ & $\begin{array}{l}\text { VEGETACIÓN } \\
\text { POTENCIAL }\end{array}$ \\
\hline \multirow{8}{*}{ 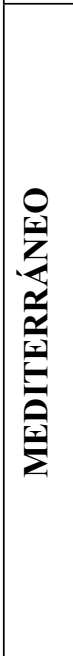 } & $\begin{array}{l}\text { Mediterráneo } \\
\text { Hiperdesértico } \\
\text { Oceánico (M.H.O.) } \\
\text { (1) }\end{array}$ & $\begin{array}{l}\text { M.H.O. Inframediterráneo } \\
\text { Ultrahiperárido }\end{array}$ & 1 & ALCA \\
\hline & $\begin{array}{l}\text { Mediterráneo } \\
\text { Desértico }\end{array}$ & $\begin{array}{l}\text { M.D.O. Inframediterráneo } \\
\text { Hiperárido }\end{array}$ & 5 & $\mathrm{ALCA}^{(1)}, \mathrm{DESA}^{(4)}$ \\
\hline & $\begin{array}{l}\text { Oceánico (M.D.O.) } \\
\text { (24) }\end{array}$ & $\begin{array}{l}\text { M.D.O. Termomediterráneo } \\
\text { Hiperárido }\end{array}$ & 1 & DESA \\
\hline & & M.D.O. Inframediterráneo Árido & 9 & $\operatorname{DESA}^{(7)}, \mathrm{MACO}^{(2)}$ \\
\hline & & M.D.O. Termomediterráneo Árido & 9 & $\mathrm{MACO}^{(5)}, \mathrm{DESA}^{(4)}$ \\
\hline & $\begin{array}{r}\text { Mediterráneo Xérico } \\
\text { Oceánico (M.X.O.) }\end{array}$ & $\begin{array}{l}\text { M.X.O. Inframediterráneo } \\
\text { Semiárido }\end{array}$ & 4 & $\mathrm{CHAPA}^{(3)}, \mathrm{MACO}^{(1)}$ \\
\hline & (37) & $\begin{array}{l}\text { M.X.O. Termomediterráneo } \\
\text { Semiárido }\end{array}$ & 28 & CHAPA \\
\hline & & M.X.O. Mesomediterráneo Semiário & 5 & PIME \\
\hline \multirow{4}{*}{ 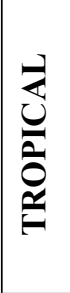 } & Mediterráneo & M.P.O. Termomediterráneo Seco & 2 & CHAPA, PIME \\
\hline & $\begin{array}{l}\text { Pluviestacional } \\
\text { Oceánico (M.P.O.) } \\
\text { (3) }\end{array}$ & M.P.O. Mesomediterráneo Seco & 1 & ENPE \\
\hline & Tropical & T.H. Termotropical Ultrahiperárido & 2 & DEMI \\
\hline & $\begin{array}{l}\text { Hiperdesértico } \\
\text { (T.H.) (4) }\end{array}$ & T.H. Mesotropical Ultrahiperárido & 2 & DEMI \\
\hline
\end{tabular}


Cuadro 5.- Continuación.

\begin{tabular}{|c|c|c|c|c|}
\hline $\mathrm{MB}$ & BIOCLIMA & ISOBIOCLIMA & $\mathrm{NE}$ & $\begin{array}{c}\text { VEGETACIÓN } \\
\text { POTENCIAL }\end{array}$ \\
\hline \multirow{19}{*}{ 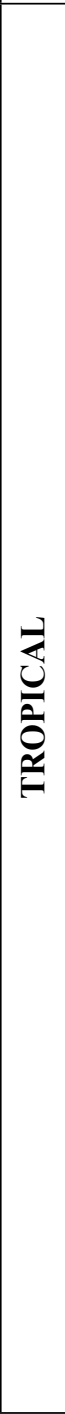 } & \multirow{4}{*}{$\begin{array}{l}\text { Tropical Desértico } \\
\text { (T.D.) (138) }\end{array}$} & T.D. Termotropical Hiperárido & 27 & $\mathrm{DESA}^{(23)}, \mathrm{DEMI}^{(4)}$ \\
\hline & & T.D. Mesotropical Hiperárido & 11 & DEMI \\
\hline & & T.D. Termotropical Árido & 91 & $\begin{array}{l}\operatorname{DESA}^{(70)}, \operatorname{DEAF}^{(11)} \\
\operatorname{MAES}^{(4)}, \mathrm{DEMI}^{(2)}, \\
\operatorname{DECR}^{(2)}, \mathrm{MESQ}^{(2)}\end{array}$ \\
\hline & & T.D. Mesotropical Árido & 9 & $\begin{array}{l}\mathrm{DEMI}^{(4)}, \mathrm{DECR}^{(3)} \\
\mathrm{DESA}^{(1)}, \mathrm{DEAF}^{(1)}\end{array}$ \\
\hline & \multirow[t]{8}{*}{$\begin{array}{c}\text { Tropical Xérico } \\
\text { (T.X.) (290) }\end{array}$} & T.X. Infratropical Semiárido & 9 & $\begin{array}{l}\mathrm{BOES}^{(6)}, \mathrm{MAES}^{(2)} \\
\mathrm{MESQ}^{(1)}\end{array}$ \\
\hline & & T.X. Termotropical Semiárido & 82 & $\begin{array}{l}\text { BOES }^{(33)}, \operatorname{MAES}^{(27)}, \\
\text { MESQ }^{(21)}, \text { DESA }^{(1)}\end{array}$ \\
\hline & & T.X. Mesotropical Semiárido & 4 & $\begin{array}{l}\operatorname{MAES}^{(2)}, \mathrm{BOES}^{(1)}, \\
\operatorname{MESQ}^{(1)}\end{array}$ \\
\hline & & T.X. Infratropical Seco & 95 & $\mathrm{BTCA}^{(85)}, \mathrm{BOES}^{(2)}$ \\
\hline & & & & $\mathrm{MANG}^{(3)}, \mathrm{MESQ}^{(5)}$ \\
\hline & & T.X. Termotropical Seco & 97 & $\begin{array}{l}\mathrm{BTCA}^{(84)}, \mathrm{MESQ}^{(11)}, \\
\mathrm{ENCA}^{(1)}\end{array}$ \\
\hline & & T.X. Mesotropical Seco & 3 & BTCA \\
\hline & & T.P. Infratropical Subhúmedo & 71 & $\begin{array}{l}\operatorname{BTSP}^{(63)}, \mathrm{MESQ}^{(3)}, \\
\mathrm{ENCA}^{(2)}, \mathrm{BOES}^{(1)}, \\
\mathrm{BTPE}^{(1)}, \mathrm{PALM}^{(1)}\end{array}$ \\
\hline & \multirow[t]{6}{*}{\begin{tabular}{|l} 
Tropical \\
Pluviestacional \\
(T.P.) (193)
\end{tabular}} & T.P. Termotropical Subhúmedo & 66 & $\begin{array}{l}\mathrm{BTSP}^{(53)}, \mathrm{ENCA}^{(8)}, \\
\mathrm{MESQ}^{(3)}, \mathrm{BTPE}^{(1)}, \\
\mathrm{BTCA}^{(1)},\end{array}$ \\
\hline & & T.P. Mesotropical Subhúmedo & 4 & $\mathrm{BTSP}^{(3)}, \mathrm{ENCA}^{(1)}$ \\
\hline & & T.P. Infratropical Húmedo & 19 & $\mathrm{BTSP}^{(10)}, \mathrm{BTPE}^{(9)}$ \\
\hline & & T.P. Termotropical Húmedo & 25 & $\begin{array}{l}\operatorname{BTSP}^{(13)}, \mathrm{BTPE}^{(10)} \\
\mathrm{ENCA}^{(1)}, \operatorname{PITR}^{(1)}\end{array}$ \\
\hline & & T.P. Infratropical Hiperhúmedo & 5 & BTPE \\
\hline & & T.P. Termotropical Hiperhúmedo & 3 & $\mathrm{BTSP}^{(2)}, \mathrm{BTPE}^{(1)}$ \\
\hline & $\begin{array}{l}\text { Tropical Pluvial } \\
\text { (T.PL.) (1) }\end{array}$ & T.PL. Infratropical Hiperhúmedo & 1 & BTPE \\
\hline
\end{tabular}

El resto de las estaciones diagnosticadas (626) se corresponden con el Macrobioclima Tropical, cuya distribución abarca la vertiente oriental y el cuadrante suroccidental de la península de Baja California y todo el resto de la vertiente pacífica 
Macías Rodríguez et al.: Clasificación bioclimática de la vertiente del Pacífico mexicano

continental desde Sonora hasta la frontera guatemalteca. Los bioclimas registrados y el número de isobioclimas son los siguientes: Tropical Hiperdesértico (2), T. Desértico (4), T. Xérico (6), T. Pluviestacional (7) y T. Pluvial (1).

En la porción nororiental de Baja California aparece el bioclima Tropical Hiperdesértico con presencia de los pisos Termotropical y Mesotropical, ambos U1trahiperáridos, y cuya vegetación potencial es el Desierto Microfilo (DEMI). Las cuatro estaciones representativas se localizan en el noreste de la Península de B.C., coincidiendo con el Desierto de San Felipe o del Colorado, la región más árida de Norteamérica (MacMahon y Wagner, 1985; Peinado et al., 2006).

El bioclima Tropical Desértico se presenta en la región denominada Delta del Río Colorado tanto en la parte bajacaliforniana como en la sonorense, en el oeste de Sonora y en Baja California Sur. En él se reconocen los pisos Termotropical y Mesotropical con ombrotipos Hiperárido y Árido; de forma particular el termotipo Termotropical muestra una mayor variación de la vegetación potencial, pudiendo reconocerse las formaciones ALCA, DEMI y DESA; el ombrotipo Árido, presente mayormente en Baja California Sur y en el norte y oeste de Sonora, se corresponde principalmente con el Desierto Arbosufrutescente (DEAF) y con el Desierto Crasicaulescente (DECR). Puntualmente en áreas favorecidas topográficamente, pueden aparecer algunas otras formaciones como DESA, Matorral Espinoso (MAES), Mesquital (MESQ), tanto en el termotipo Termotropical como en el Mesotropical.

Los bioclimas Tropical Xérico y Pluviestacional son los de mayor distribución a lo largo de la vertiente del Pacífico. El Tropical Xérico existe bajo los ombrotipos Semiárido y Seco, estando el primero presente en el sur de Sonora y de Baja California Sur y en el centro y sur de Sinaloa, asociado principalmente a planicies donde la vegetación potencial corresponde a los denominados Bosque Espinoso (BOES), MAES y MESQ. La otra zona con ombrotipo Semiárido se localiza más al sur, en la cuenca inferior de los ríos Balsas-Tepalcatepec (Guerrero y Michoacán) y en la región del Istmo de Tehuantepec (Oaxaca y Chiapas). El ombrotipo Seco se corresponde con el dominio del Bosque Tropical Caducifolio (BTCA), presente bajo los termotipos Infratropical, Termotropical y Mesotropical inferior. Se distribuye principalmente a lo largo de laderas, barrancas y piedemontes de las SMO, SMS y el EVT, así como los territorios del tramo inferior de la depresión del Balsas-Tepalcatepec.

El bioclima Tropical Pluviestacional se distribuye de manera discontinua desde el sur de Sinaloa hasta Chiapas. Está vinculado a territorios con precipitaciones mayores que las correspondientes al bioclima Tropical Xérico, las cuales se ven favorecidas por los flujos de vientos más húmedos que descargan sobre sierras, cañadas y piedemontes directamente orientados hacia ellos. Los ombrotipos represen- 
tados son el Subhúmedo, Húmedo e Hiperhúmedo. El primero es el más amplio y se relaciona con las formaciones de Bosque Tropical Subperennifolio (BTSP) presente tanto en el piso Infratropical como en el Termotropical. El ombrotipo Húmedo está asociado a las planicies costeras y laderas de los estados de Chiapas y en menor medida de Oaxaca (termotipos Infra- y Termotropical), y se corresponde con las formaciones de BTSP. El ombrotipo Hiperhúmedo se relaciona con los BTPE emplazados en aquellas zonas más húmedas y de menor latitud de la franja costera de Chiapas; esta situación se asocia a la zona de transición entre los bioclimas Tropical Pluviestacional y Tropical Pluvial. Para este último solo se registró una estación, San Jerónimo (Chiapas), localizada en el piso Infratropical Hiperhúmedo, próxima a la frontera con Guatemala.

Vegetación potencial

La clasificación bioclimática utilizada pone de relieve la secuencia real en la distribución de la vegetación potencial. En el área de estudio, los bioclimas tropicales más áridos (Desértico e Hiperdesértico) se extienden a través de una franja latitudinal de unos $650 \mathrm{~km}$ que podría denominarse como la región Larrea tridentata-Parkinsonia microphylla, dado que ambas especies son los mejores indicadores de dichos bioclimas. La ausencia de ambos taxones en las zonas áridas de la costa del Pacífico delimita con precisión la estrecha franja de macrobioclima Mediterráneo existente en El Vizcaíno. En las zonas desérticas relativamente más húmedas (ombrotipo Árido), P. microphylla junto con varias cactáceas columnares, es un componente típico de la vegetación potencial zonal del DEAF y del DECR; por el contrario, en áreas de menor precipitación (ombrotipo Hiperárido) su presencia se restringe a comunidades azonales de suelos húmedos correspondiente a las asociaciones Hymenocleo salsolae-Daleetum spinosae (Peinado et al., 1995a) y Parkinsonio microphyllae-Olneyetum tesotae (Peinado et al., 2006) o a biotopos rocosos del DESA. Larrea tridentata domina o codomina en todo tipo de comunidades zonales, y su área de distribución marca, en la zona de estudio, las fronteras de los bioclimas Tropical Desértico y Tropical Xérico; su desaparición al sur de Guaymas coincide con la zona de transición entre la vegetación de desiertos áridos y los matorrales espinosos semiáridos (Shreve, 1951; Turner et al., 1995), es decir, donde comienza la zona de isobioclima Tropical Xérico Termotropical Semiárido. Esta banda de transición gradual entre los desiertos y los bosques tropicales caducifolios es ocupada por matorrales y bosques espinosos considerados como zonoecotono entre los zonobiomas II y III (Walter, 1985) y coincide con la transición entre los bioclimas Tropical Desértico y Tropical Xérico. Así, desde Guaymas hacia el 
Macías Rodríguez et al.: Clasificación bioclimática de la vertiente del Pacífico mexicano

norte, hasta el río Sonora, las formaciones de bosques espinosos dominadas por especies tales como Acacia cymbispina, Ceiba acuminata, Haematoxylum brasiletto, Ipomoea arborescens, Pachycereus pecten-aboriginum y Pithecellobium sonorae, aparecen confinadas en los lechos de arroyos y en los valles ligeramente más húmedos, lo que representa una introgresión de la vegetación xérica en zonas de bioclima Tropical Desértico. Al sur de Guaymas desaparecen las plantas más típicas de los desiertos, y los árboles y arbustos espinosos anteriores comienzan a dominar en el paisaje, mientras que subsisten pequeñas áreas de vegetación desértica (DEAF) en los hábitats más áridos, como laderas de solana con suelos poco evolucionados. Para una descripción más detallada de ese ecotono véase Búrquez et al. (1999) y Sánchez-Mejía et al. (2007).

Un cactus columnar, Pachycereus pecten-aboriginum, y el palo blanco, $L y$ siloma divaricatum, son los mejores indicadores del bioclima Tropical Xérico, para el que se reconocen cuatro tipos de vegetación potencial: MAES, BOES, MESQ y BTCA. En términos geobotánicos clásicos, estos tipos pueden ser descritos como formaciones de Hiemilignosa, que se presentan con la estructura arbustiva de las Hiemifruticeta (ombrotipo Semiárido Inferior: MAES), la estructura intermedia arbustivo-arborescente de la transición Hiemifruticeta-Hiemisilva (ombrotipo Semiárido Superior: BOES, MESQ), o en las zonas más húmedas con ombroclima Seco, con la típica estructura forestal más o menos densa de las Hiemisilva (BTCA).

Con ombroclima Semiárido, las plantas dominantes son principalmente arbustos (en los MAES) o pequeños árboles (en los BOES), en ambos casos espinosos $\mathrm{y}$ verdes tras las lluvias estivales, cuya estructura se asemeja a la "caatinga" del noreste de Brasil, al "semidesierto" venezolano de cactáceas y fanerófitos espinosos o a los matorrales espinosos de Madagascar (Walter, 1985; Takhtajan, 1986). Predominan diferentes especies de Acacia, Caesalpinia, Cassia, Mimosa, entre otras leguminosas, lo que pone de manifiesto las relaciones pantropicales de las formaciones espinosas del Nuevo y del Viejo Mundo, las cuales están relacionadas con acontecimientos paleoclimáticos y paleogeográficos (Peinado et al., 2009). Nuestro análisis de la región de Los Cabos de Baja California revela estrechos lazos florísticos entre las comunidades espinosas y los bosques tropicales caducifolios que permiten unirlos en una sola clase fitosociológica: Pachycereo pecten-aborigini-Lysolometea divaricati (Peinado et al., 2008).

De los retazos de vegetación natural que todavía persisten en las planicies aluviales del noroeste, coincidiendo con los deltas de los ríos Yaqui, Batacosa, Mayo y Fuerte, se deduce que la vegetación azonal se corresponde con los BOES dominados por el mezquite (Prosopis glandulosa var. torreyana), y donde Parkinsonia 
praecox y varias especies de Bursera son acompañantes habituales en el dosel arbóreo (MESQ). El comportamiento freatófilo de esos bosques sobresale en abril, cuando los mezquites están en plena foliación y forman grandes manchas verdes que contrastan con la vegetación agostada (MAES, BOES) de las laderas. La estructura básica de los MAES y los BOES está dominada por pequeños árboles multicaules y por arbustos altos xeromórficos, espinosos, microfilos y caducifolios por sequía, además de suculentas; buena parte de estos componentes se comparten con la vegetación de los desiertos del DEAF y del DECR. En los BOES la altura de los árboles es mayor que en los MAES y algunas plantas que son arbustivas en estos últimos aparecen como árboles en los BOES (por ejemplo: Erythrina flabelliformis, Haematoxylum brasiletto y Bonellia macrocarpa ssp. pungens). El dosel de árboles pequeños xeromórficos y microfilos presenta una altura que oscila entre 4 m (MAES) y $10 \mathrm{~m}$ (BOES); por el contrario ciertas cactáceas columnares como Stenocereus thurberi, Pachycereus pecten-aboriginum, Cephalocereus purpusii y algunos nopales arborescentes emergen notablemente sobre los arbustos en los MAES e igualan las copas de los árboles en los BOES.

En la Figura 3 se presenta un transecto idealizado de la vegetación entre el Océano Pacífico y la SMO a la altura del paralelo $27^{\circ} 30^{\prime} \mathrm{N}$, en el que se incluyen áreas de media montaña adyacentes a la zona de estudio. En las zonas de planicies próximas a la costa, bajo el bioclima Tropical Xérico con ombrotipos Árido y Semiárido, se desarrolla el MAES y el BOES; en territorios un poco más elevados domina

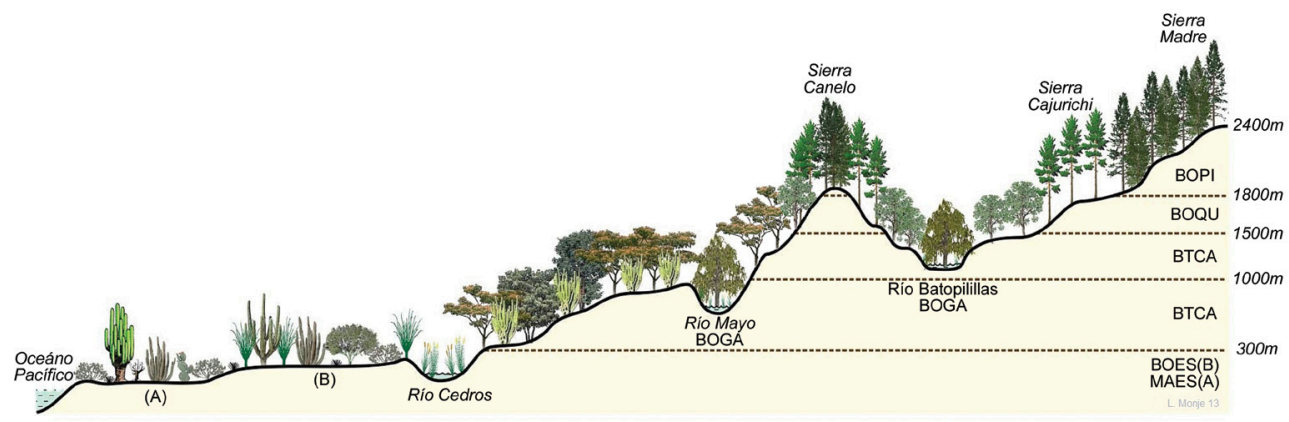

Fig. 3. Distribución esquemática de los principales tipos de vegetación en la Sierra Madre Occidental. Sección transversal a lo largo del eje de los ríos Mayo y Batopilillas, desde la Bahía de Yavaros, Sonora, a la Cascada de Basaseachic Parque Nacional, Chihuahua. Abreviaturas: BOPI, Bosque de Pinus; BOEN, Bosque de Encino; BTCA, Bosque Tropical Caducifolio; BOGA, Bosque de Galería; BOES, Bosque Espinoso; MAES, Matorral Espinoso. 
Macías Rodríguez et al.: Clasificación bioclimática de la vertiente del Pacífico mexicano

el ombrotipo Seco, donde se presenta el BTCA, alcanzando una cota aproximada de $1500 \mathrm{~m}$. Posteriormente, ya en el piso Mesotropical Subhúmedo (bioclima Tropical Pluviestacional), hacen presencia las formaciones de Bosque de pino (BOPI) y de Bosque de encino (BOEN).

Con ombrotipo Seco, la vegetación dominante son los bosques tropicales caducifolios (BTCA), que corresponden al Zonobioma II de Walter (1985), presentes en otros territorios tropicales análogos. A lo largo de la vertiente del Pacífico Norte se distribuyen formando una franja desde la cuenca alta del río Mayo, en Sonora meridional, hasta Costa Rica (Brown et al., 1998), con una anchura media de unos 50 km (Gentry, 1995). El relativo aislamiento de los BTCA del interior de Sonora con respecto a otros BTCA mesoamericanos ha favorecido la aparición de un elevado número de géneros endémicos, lo que hace que estos bosques sean florísticamente más diversos que sus vicariantes de Mesoamérica (Trejo y Dirzo, 2002; Rzedowski y Calderón, 2013). Además de habitar en la vertiente del Pacífico, los BTCA se extienden por el centro de México y, con algunas diferencias florísticas, aparecen también en la provincia Sanlucana de Baja California (Peinado et al., 1997a, 2008) y en tres grandes zonas de la vertiente atlántica mexicana (Rzedowski, 1978; Anónimo, 1997).

El paisaje de toda la zona ocupada por los BTCA (como también sucede en el área potencial de los BOES) está hoy muy alterado a causa de los cultivos, la ganadería extensiva y la masiva invasión del pasto africano Pennisetum ciliare (Búrquez et al., 2002). Pese a ello, los BTCA resisten en sus límites septentrionales favorecidos por el relieve extraordinariamente abrupto de la SMO. En su área de distribución la composición específica varía enormemente de una localidad a otra debido a la gran extensión de esos bosques y a las diferencias ecológicas provocadas por la exposición del terreno, las pendientes, la elevación altitudinal, el sustrato, la profundidad del suelo y otros factores de índole local poco conocidos, que pudieran estar íntimamente relacionados con la disponibilidad de agua para la germinación y el crecimiento juvenil de los árboles (Balvanera et al., 2001). En cualquier caso es destacable la presencia reiterada de representantes de los géneros Amphipterygium, Bursera, Caesalpinia, Ceiba, Cordia, Lysiloma, Pseudobombax, entre otros.

En las zonas de transición BOES y BTCA se entremezclan, aunque cuando dominan los últimos se distinguen por su mayor altura, su follaje más latifolio, la escasez o ausencia de plantas espinosas y suculentas, y por la fuerte penetración de especies, géneros y familias de distribución tropical, incluyendo orquídeas y bromeliáceas epífitas. A diferencia de lo que sucede en los BOES, en los BTCA las cactáceas arborescentes antes mencionadas son más bajas que el dosel arbóreo quedando 
enmascaradas en él. Dos palmas de tamaño mediano, Acrocomia aculeata y Sabal rosei, que nunca se presentan en las áreas de ombroclima Semiárido, son buenas bioindicadoras del ombrotipo Seco, siendo favorecida su presencia por los fuegos vinculados a la actividad pecuaria.

Enterolobium cyclocarpum, Astronium graveolens y Brosimum alicastrum son los mejores bioindicadores del BTSP y su aparición se vincula al bioclima Tropical Pluviestacional de los pisos Infratropical o Termotropical al menos Subhúmedo. Este bosque presenta una estructura y altura intermedia entre los BTCA y los BTPE. En situaciones de mayor pluviosidad (Bioclimas Pluviestacional o Pluvial con ombrotipo al menos Hiperhúmedo) el BTPE constituye la vegetación potencial, siendo el tipo de vegetación más estructurado y complejo en el área de estudio. A medida que la precipitación aumenta, en los bosques caducifolios comienzan a hacerse cada vez más visibles los árboles perennifolios. Dado que para la misma latitud la vertiente Atlántica mesoamericana es más húmeda que la Pacífica, los BTPE tienen mayor presencia en la primera; su representación en la vertiente Pacífica está relegada a los enclaves más ombrófilos próximos a la costa chiapaneca. En el resto de dicha vertiente los BTCA y los BTSP son los representantes mayoritarios de la vegetación potencial dominante desde Sinaloa meridional hasta Chiapas (Leopold, 1950; Rzedowski, 1978).

Debido a la naturaleza transicional de los BTSP, su estructura está dominada por un dosel arbóreo en el que coexisten árboles perennifolios y caducifolios. En situaciones óptimas de conservación, el dosel arbóreo alcanza una altura de 30-40 m con tres estratos arbóreos bien diferenciados. El superior está formado por megafanerófitos emergentes tales como Enterolobium cyclocarpum, Brosimum alicastrum, Astronium graveolens y Conzattia multiflora, que sobresalen sobre dos estratos más continuos en los que se entremezclan caducifolios y perennifolios. La proporción de árboles caducifolios es mayor en el estrato medio, mientras que en el inferior dominan principalmente los perennifolios microfilos. Algunas higueras estranguladoras del género Ficus sobresalen entre las lianas, las cuales forman un estrato difuso y enmarañado en los huecos dejados por los árboles caídos. En las llanuras y colinas poco abruptas, la palmera Attalea cohune codomina en el estrato superior e incluso llega a formar palmares naturales (PALM) en suelos arenosos bien drenados, los que en la actualidad han sido sustituidos en buena parte por plantaciones de Cocos nuccifera, Mangifera indica y Carica papaya. Como sucede con otras palmas tropicales, $A$. cohune es resistente a los incendios y forma comunidades secundarias en lugares fuertemente alterados por actividades humanas.

La adscripción bioclimática de los pinares, encinares y sus bosques mixtos, formaciones representativas de la vegetación clímax más extendida en las montañas 
Macías Rodríguez et al.: Clasificación bioclimática de la vertiente del Pacífico mexicano

de México (Rzedowski, 1978), está vinculada mayoritariamente al bioclima Tropical Pluviestacional, aunque también se presentan en el Bioclima Tropical Xérico, especialmente bajo el ombrotipo Seco. Desde el punto de vista térmico sus formaciones ocupan mayoritariamente el gradiente altitudinal entre el termotipo Mesotropical y el Orotropical (Medina et al., 2012; Giménez de Azcárate et al., 2013). Sin embargo, en la vertiente del Pacífico dichas formaciones descienden a cotas bajas (Rzedowski y McVaugh, 1966) representativas del termotipo Termotropical. En nuestra opinión y tras haber analizado los bosques mencionados de media montaña de ambas Sierras Madres, éstos constituyen la vegetación potencial climácica en el piso Mesotropical cuando el ombrotipo es al menos Subhúmedo. En altitudes inferiores, bajo condición Termotropical, la vegetación potencial climácica corresponde a bosques que alternan en función de la cuantía de las precipitaciones: caducifolios (ombrotipo Seco), subperennifolios (ombrotipo Subhúmedo y Húmedo) y perennifolios (ombrotipos Hiperhúmedo y Ultrahiperhúmedo). En tales condiciones, los ENCA y los PITR constituyen comunidades edafoxerófilas que ocupan suelos poco profundos, ricos en gravas, evolucionados a partir de rocas derivadas de cenizas volcánicas, lateritas, riolitas, andesitas o basaltos, pobres en nutrientes y ricos en metales pesados; en general se disponen sobre pendientes muy inclinadas donde la fuerte escorrentía favorece la lixiviación gravitacional de los escasos nutrientes. Sarukhán (2005) ha subrayado el factor edáfico como condicionante esencial de los encinares y los pinares tropicales (Termotropicales en el contexto de este trabajo). De acuerdo con nuestras observaciones, todavía provisionales, mientras que los ENCA aparecen como comunidades edafoxerófilas ligadas a las áreas ocupadas por los BTCA (ombrotipo Seco), los PITR y los bosques mixtos lo hacen igualmente sobre biotopos azonales como los descritos, pero donde los BTSP y en menor medida los BTPE constituyen la vegetación potencial climácica (ombrotipo al menos Subhúmedo). Pese a la extraordinaria diversidad del género Quercus en México, ésta desciende drásticamente de los territorios húmedos y subhúmedos del piso Mesotropical a los secos termotropicales, donde la especie preferente es Quercus resinosa a la que pueden acompañar otros encinos como $Q$. glaucescens, $Q$. glaucoides y otros árboles del BTCA.

Algunas de las estaciones muestreadas están situadas dentro de áreas muy extensas de manglar. En esos casos, aunque se trate de vegetación azonal, se han considerado a los manglares como la vegetación potencial. Por el norte del área de estudio, los manglares (MANG) comienzan a aparecer en el piso Termotropical del Golfo de California, en Bahía de los Ángeles (29 $\left.05^{\prime} \mathrm{N}\right)$, donde se encuentra su límite de distribución septentrional en el reino Neotropical (Peinado et al., 2008). Los MANG aparecen en todas las costas mexicanas excepto en las mediterráneas del 
noroeste de Baja California, donde son reemplazados por las comunidades arbustivas y herbáceas de saladares de la clase Limonio californici-Frankenietea salinae (Peinado et al., 1995b). En todas las localidades muestreadas los manglares presentan una zonación similar a la observada en otras zonas norteamericanas, caribeñas y mesoamericanas (Peinado et al., op cit.).

\section{DISCUSIÓN}

Los resultados aquí plasmados ilustran el escenario geobotánico de un territorio donde los gradientes y ritmos climáticos condicionan y moldean la vegetación. La heterogeneidad ecológica del área considerada permitió el reconocimiento de una secuencia de tipos de vegetación que se sustituyen latitudinalmente o altitudinalmente como respuesta a las condiciones climáticas cambiantes, dando lugar a los denominados pisos o cinturas de vegetación. Este remplazo adaptativo a sucesivos pisos bioclimáticos se delimita en función de termotipos y ombrotipos.

A excepción de algunas estaciones próximas a la cota de $1000 \mathrm{~m}$ situadas en el noroeste de Baja California, y donde los inviernos más severos favorecen las heladas, toda la zona analizada está libre de ellas por lo que la vegetación está más condicionada por el ritmo y el conjunto de las precipitaciones que por las bajas temperaturas. En respuesta al ritmo de lluvias, en la zona de estudio, al igual que en el resto de México, aparecen dos macrobioclimas: Mediterráneo y Tropical. El macrobioclima Mediterráneo se caracteriza por presentar inviernos húmedos y veranos secos con un mínimo de dos meses consecutivos con precipitación inferior al doble de la temperatura media $(\mathrm{Pm}<2 \mathrm{Tm})$ (Bagnouls y Gaussen, 1953). Esta caracterización permite discriminar la mayoría de las estaciones tropicales frente a las mediterráneas en toda Norteamérica (Macías, 2009; Peinado et al., 2010, 2011) y, por ende, en la zona de estudio.

Las zonas tropicales se caracterizan por presentar una estación de lluvias coincidente con el período más cálido del año, en contraste con otro de sequía de duración variable coincidente con la temporada de temperaturas relativamente más bajas (Walter, 1985). En función de la duración, cuantía y eficiencia de las precipitaciones, esta última condicionada por las temperaturas, es posible identificar los diferentes bioclimas. Para el territorio Tropical del área de estudio, su amplio rango latitudinal lleva asociado una alta complejidad climática, reflejada en la presencia de los cinco bioclimas Tropicales que se identifican en la Tierra, y en las diferentes estructuras de las formaciones vegetales asociadas. 
Macías Rodríguez et al.: Clasificación bioclimática de la vertiente del Pacífico mexicano

El bioclima Tropical Hiperdesértico se corresponde con una zona de transición con influencia tanto del régimen Mediterráneo como Tropical; la presencia de Larrea tridentata, entre otros taxones, confirma la adscripción Tropical de su vegetación (Peinado et al., 2010, 2011).

Al sur de Bahía Asunción (Baja California Sur), coincidiendo con la aparición de los manglares, inicia el bioclima Tropical Desértico, predominante en el resto de la península excepto en algunas estaciones Tropical Xéricas y Tropical Hiperdesérticas. Las primeras están situadas en zonas altas (como Ejido San Matías, a $968 \mathrm{~m}$ s.n.m., que es la única estación Tropical Xérica del estado de Baja California) o en el extremo sur de la península, en la provincia biogeográfica Sanlucana, que por su posición meridional y más oriental con respecto al resto de la península, están más influidas por las precipitaciones de origen convectivo y por los huracanes del Pacífico. En las estaciones con ombrotipo Árido, localizadas en el pasillo formado por los valles aledaños a la desembocadura del Río Colorado, noreste de Baja California (Colonia Mariana, Cerro Prieto, Colonia San Pedro Mártir y Colonia Juárez), el ritmo estacional de precipitación resulta doblemente influido tanto por las invernales que penetran hacia el interior a través de la Ruta Sonorense, como por las precipitaciones de fin del verano y principio de otoño. Así presentan un régimen bimodal de precipitaciones que difiere claramente del régimen unimodal que prevalece en el resto de la zona analizada. La escasez de lluvias y la influencia de los regímenes pluviales que afectan a estas zonas de transición mediterránea-tropical provocan unos ritmos de precipitación erráticos imposibles de ajustar a un modelo numérico.

Por ello la presencia de comunidades con Larrea tridentata es determinante para que, en esas zonas transicionales, una estación sea considerada como Tropical con independencia de que la clasificación utilizada la incluya en el macrobioclima Mediterráneo. Una situación análoga ocurre con otras dos localizadas en el Desierto Central (Chapala y San Agustín), que de acuerdo con la clasificación de Rivas-Martínez las ubica como Mediterráneo-Desérticas. Finalmente todas estas estaciones fueron registradas como tropicales, ya que su vegetación está dominada por especies crasicaulescentes y por arbustos xerofíticos (Peinado et al., 1995c, 2005), consideradas especies propias de la región Xerofítico-Mexicana (Rzedowski, 1978) y características de Prosopido torreyanae-Fouquieretea splendentis, una clase fitosociológica que encierra a la vegetación de los desiertos neotropicales norteamericanos (Rivas Martínez, 1997; Peinado et al., 2005).

En México continental, las estaciones septentrionales y más áridas, en su mayoría sonorenses (salvo Ahome y Mochicahui, en Sinaloa) presentan bioclima Tropical Desértico e Hiperdesértico, el primero de los cuales se extiende de forma casi continua entre San Luis Río Colorado y la cuenca septentrional del río Yaqui, 
cerca de Guaymas. Al sur de esta localidad, coincidiendo con un incremento de las precipitaciones, comienza un grupo continuo de estaciones Tropical Xéricas en Sonora, Sinaloa y norte de Nayarit. En la costa central de Nayarit, donde el EVT alcanza la costa Pacífica, aparecen las primeras estaciones con bioclima Tropical Pluviestacional que, desde allí y de forma más o menos continua, se extienden hacia el sur, alternando, en las áreas que quedan en sombra de lluvias de los sistemas montañosos, con algunas estaciones de bioclima Tropical Xérico. Solo una estación, San Jerónimo (Chiapas), presenta bioclima Tropical Pluvial; si bien no es la que recoge mayor cantidad de precipitación $(4126.5 \mathrm{~mm})$, ésta se reparte de forma bastante uniforme a lo largo del año, lo que permite su encuadre en el bioclima Tropical Pluvial, a diferencia de Santo Domingo (Chiapas) $(4768.4 \mathrm{~mm})$, donde la precipitación presenta un pequeño descenso en el mes de febrero, responsable de su adscripción dentro del bioclima Tropical Pluviestacional.

Aunque los valores de precipitación y temperatura para cada estación están muy influidos por factores locales como la altitud, exposición y orografía, en la zona de estudio se observan varios gradientes latitudinales y longitudinales. En primer lugar, hay un claro incremento térmico hacia el sur. Por el contrario, el índice de continentalidad (Ic), disminuye latitudinalmente porque la estabilidad térmica aumenta en dirección al ecuador. La precipitación anual (P) en la zona de estudio oscila entre un mínimo de $41 \mathrm{~mm}$ (La Ventana, Baja California, probablemente el menor registro de Norteamérica), situada en el extremo septentrional de la zona de estudio, y el máximo de Santo Domingo anteriormente comentado, y que a su vez constituye el tercer registro más alto de la costa oriental del Pacífico Norte (Macías, 2009). En Baja California, mientras que la media de precipitaciones invernales para las estaciones de la costa del Pacífico es de $101 \mathrm{~mm}$, para las del Golfo de California es cinco veces menor. Inversamente, la media de precipitaciones estivales para las estaciones del Golfo es cuatro veces mayor que la registrada en las del Pacífico.

A nivel del mar en la costa del Pacífico, Tijuana recibe $250 \mathrm{~mm}$ anuales. Esta cifra disminuye rápidamente hacia el sur a lo largo de la costa. En El Socorro, donde los chaparrales mediterráneos comienzan a declinar en beneficio de la vegetación desértica, recibe solo la mitad. Desde esta localidad hacia el sur la precipitación disminuye aún más, con los mínimos registrados al norte y al sur de la península del Vizcaíno, donde aún se registran lluvias invernales significativas, dentro del contexto de escasez general de estas áreas desérticas. Al sur de la península de El Vizcaíno, superadas Punta Eugenia y Bahía Tortugas, cesa el predominio de la precipitación invernal. Al sur de Bahía Magdalena, en plena latitud de los alisios y de los huracanes otoñales, aumentan tanto las precipitaciones de verano como las de otoño con 
un incremento porcentual cada vez mayor según se avanza hacia el sur. Aunque estos gradientes norte-sur son muy notables, resultan oscurecidos por el espectacular gradiente oeste-este motivado por la influencia del Pacífico. Por ejemplo, entre las estaciones Ejido Valle de La Trinidad, en la vertiente pacífica, y La Ventana, en la del Golfo, separadas tan solo por $70 \mathrm{~km}$, la precipitación invernal desciende de 124 $\mathrm{mm}$ a poco menos de $14 \mathrm{~mm}$.

Las variaciones en la precipitación y la temperatura a lo largo del área de estudio están reguladas por factores latitudinales, longitudinales, regionales y locales. El hecho de que prácticamente la totalidad del territorio analizado libre de heladas, es responsable de que la vegetación esté condicionada principalmente por la cantidad y el ritmo de las precipitaciones. La acción distorsionadora del modelo macroclimático a escala regional es provocada por los grandes sistemas montañosos (SMO, EVT, SMS y SMCh), responsables de lluvias orográficas en las laderas de barlovento y fuertes sombras de lluvia en las de sotavento.

Para obtener una mejor correlación entre los bioclimas y la vegetación, se requiere llevar a cabo estudios a mayor escala, ya sea estatal o local, que permitan una equiparación más precisa y ajustada de los isobioclimas, así como seguir afinando en las relaciones clima-vegetación de cara al establecimiento y ajuste de un modelo predictivo de referencia frente a los procesos de cambio global.

\section{AGRADECIMIENTOS}

Este estudio fue financiado con fondos de un proyecto de investigación del Instituto Franklin de Investigación de Estudios Norteamericanos (Universidad de Alcalá). Los autores agradecen al profesor Dr. Francisco Alcaraz de la Universidad de Murcia la ayuda desinteresada para ejecutar el programa Bioclima, al Q.F.B. Ángel Pérez Zamora de la Universidad de Guadalajara, su colaboración en realización del mapa.

\section{LITERATURA CITADA}

Alcaraz, F. 2013. Bioclimatología con R. Universidad de Murcia. Disponible en: http://www. um.es/docencia/geobotanica/ficheros/practical.pdf

Anguiano, J., J. A. Ruiz, J. J. Alcántar, I. Vizcaíno e I. J. González. 2005. Estadísticas climatológicas básicas para el estado de Michoacán (Período 1961-2003). Libro Técnico Núm. 3. Instituto Nacional de Investigaciones Forestales, Agrícolas y Pecuarias-Centro de Investigación Regional del Pacífico Centro. Uruapan, México. 248 pp. 
Anónimo. 1995a. Síntesis de información geográfica del estado de Sonora. Información Digital. Instituto Nacional de Estadística, Geografía e Informática. Aguascalientes, México.

Anónimo. 1995b. Síntesis de información geográfica del estado de Baja California Sur. Información Digital. Instituto Nacional de Estadística, Geografía e Informática. Aguascalientes, México.

Anónimo. 1997. Imágenes digitales de la carta de uso del suelo y vegetación de México, Escala 1: 1000 000. Cubrimiento Nacional en Disco Compacto. Información Digital. Instituto Nacional de Estadística, Geografía e Informática. Aguascalientes, México.

Anónimo. 2001. Síntesis de información geográfica del estado de Baja California. Información Digital. Instituto Nacional de Estadística, Geografía e Informática. Aguascalientes, México.

Anónimo. 2002. Síntesis de información geográfica del estado de Nayarit. Información Digital Instituto Nacional de Estadística, Geografía e Informática. Aguascalientes, México.

Anónimo. 2013. Servicio Meteorológico Nacional y Comisión Nacional del Agua $<$ http:// smn.cna.gob.mx $>$ (consultado de septiembre de 2011 a septiembre de 2012).

Bagnouls, F. y H. Gaussen 1953. Saison sèche et indice xérothermique. Bull. Soc. Hist. Nat. Toulouse 88: 193-239.

Balvanera, P., G. Segura, C. Siebe y M. Martínez. 2001. Patterns of Beta diversity in a tropical dry forest. J. Veg. Sci. 13: 145-158.

Beard, J. S. 1973. The physiognomic approach. In: Whittaker, R. H. (ed.). Ordination and classification of communities. Dr. W. Junk Publishers. The Hague, Holanda. pp. 355387.

Box, E. O. 1981. Macroclimate and plant forms: an introduction to predictive modeling in phytogeography. Dr. W. Junk Publishers. The Hague, Holanda. 258 pp.

Brouillet, L. y R. D. Whetstone. 1993. Climate and physiography. In: Flora of North America Editorial Committee (eds.). Flora of North America north of Mexico. Vol. 1. Oxford University Press. New York, USA. pp. 15-46.

Brown, D. E., F. W. Reichenbacher y S. Franson. 1998. The biotic communities of North America. University of Utah Press. Salt Lake City, USA. 141 pp.

Bryson, R. A. y F. K. Hare. 1974. The climates of North America. In: Landsberg, H. E. (ed.). World survey of climatology. Elsevier. Amsterdam, Holanda. pp. 1-47.

Búrquez, A., A. Martínez-Yrízar, R. S. Felger y D. Yetman. 1999. Vegetation and habitat diversity at the southern edge of the Sonoran Desert. In: Robichaux, R. H. (ed.). Ecology of Sonoran Desert plants and plant communities. University of Arizona Press. Tucson, USA. pp. 36-67.

Búrquez, A., M. E. Miller y A. Martínez-Yrízar. 2002. Mexican grasslands, thornscrub and the transformation of the Sonoran Desert by invasive exotic buffelgrass (Pennisetum ciliare). In: Tellman, B. (ed.). Invasive exotic species in the Sonoran Region. University of Arizona Press. Tucson, USA. pp. 126-146.

Cress, J., R. Sayre, P. Comer y H. Warner. 2009. Terrestrial ecosystems. Isobioclimates of the conterminous United States: U. S. Geological Survey Scientific Investigations Map 3084, scale 1:5 000 000, 1 sheet. Disponible en http://pubs.usgs.gov/sim/3084. 
Macías Rodríguez et al.: Clasificación bioclimática de la vertiente del Pacífico mexicano

Del Río-González, S. 2005. El cambio climático y su influencia en la vegetación de Castilla y León (España). Itinera Geobot. 16: 5-534.

Douglas, M. W., R. A. Maddox y K. Howard. 1993. The Mexican monsoon. J. Climate 6: 1655-1677.

Felger, R. S. 2000. Flora of the Gran Desert and Río Colorado of Northwestern Mexico. University of Arizona Press. Tucson, USA. 673 pp.

Géhu, J. M. 2006. Dictionnaire de sociologie et synécologie végétales. J. Cramer. BerlínStuttgart, Alemania. 899 pp.

Gentry, A. H. 1995. Diversity and floristic composition of Neotropical dry forests. In: Bullock, S. H., H. A. Mooney y E. Medina (eds.). Seasonally dry tropical forests. Cambridge University Press. Cambridge, UK. pp. 146-194.

Gentry, H. S. 1942. Río Mayo plants. A study of the flora and vegetation of the Valley of the Río Mayo, Sonora. Carnegie Inst. Wash. Pub. 527: 1-328.

Giménez de Azcárate, J., M. Á. Macías y F. Gopar. 2013. Bioclimatic belts of Sierra Madre Occidental (México): A preliminary approach. Inter. J. Geobot. Research 3(1): 19-35.

González-Elizondo, M. S., M. González-Elizondo, J. A. Tena, L. Ruacho, I. L. LópezEnríquez. 2012. Vegetación de la Sierra Madre Occidental, México: una síntesis. Acta Bot. Mex. 100: 351-403.

Google Earth. 2013. vs 5.1.3533.1731. Data SIO, NOAA, U.S. Navy, NGA, GEBCO, US Dept of State Geographer.

Hamilton, W. 1971. Recognition on space photographs of structural elements of Baja California. U.S. Geological Surv. Prof. Paper 718: 1-26.

Hastings, J. R. y R. M. Turner. 1965. Seasonal precipitation regimes in Baja California, Mexico. Geogr. Ann. 47: 204-223.

Larcher, W. 2003. Physiological plant ecology. 4a. ed. Springer-Verlag. Berlin, Alemania. $513 \mathrm{pp}$.

Leopold, A. S. 1950. Vegetation zones of México. Ecology 31: 507-518.

Macías, M. Á. 2009. Estudio de las relaciones entre zonobiomas, bioclimas y vegetación en la costa del Pacífico norteamericano. Tesis Doctoral. Universidad de Alcalá. Alcalá de Henares, España. 634 pp.

MacMahon, J. A. y F. W. Wagner. 1985. The Mohave, Sonoran and Chihuahuan deserts of North America In: Evanari, M., Y. Noy-Meir y D. W. Goodall (eds.). Ecosystems of the world: Hot deserts and arid shrublands. Vol. 12. Elsevier. Amsterdam, Holanda. pp. 105-202.

Martin, P. S. y D. A. Yetman. 2000. Introduction and prospect: Secrets of a tropical deciduous forest. In: Robichaux, R. H. y D. A. Yetman (eds.). The tropical deciduous forest of Alamos. Biodiversity of a threatened ecosystem in Mexico. The University of Arizona Press. Tucson, USA. pp. 3-18.

Medina, C., F. Gopar, J. Giménez de Azcárate y A. Velázquez. 2012. Análisis bioclimático y estudio de la vegetación del transecto Pico del Tancítaro-Valle de Apatzingán, Michoacán, México. In: Mas, J. F. y G. Cuevas (comps.). Memorias XIX Reunión Internacional Geógrafos Latinoamericanos. Sociedad Latinoamericana de Percepción Remota y Sistemas de Información Espacial. Centro de Investigaciones en Geografía Ambiental, Universidad Nacional Autónoma de México. Morelia, México. pp. 293-301. 
Mosiño, P. A. y E. García. 1974. The climate of Mexico. In: Bryson, R. A. y F. K. Hare (eds.). Climates of North America. Elsevier. Amsterdam y New York. pp. 345-404.

Müller, M. J. 1982. Selected climatic data for a global set of standard stations for vegetation science. Dr. W. Junk Publ. The Hague, Holanda. 306 pp.

Peinado, M. y J. Delgadillo Rodríguez. 1990. Introducción al conocimiento fitotopográfico de Baja California (México). Studia Bot. 9: 25-39.

Peinado, M., F. Alcaraz, J. L. Aguirre y J. Álvarez. 1994a. Vegetation formations and associations of the zonobiomes along the North American Pacific coast. Vegetatio 114: 123-135.

Peinado, M., F. Alcaraz, J. Delgadillo Rodríguez e I. Aguado. 1994b. Fitogeografía de la península de Baja California, México. Anales Jard. Bot. Madrid 51(2): 255-277.

Peinado, M., C. Bartolomé, J. Delgadillo Rodríguez e I. Aguado. 1994c. Pisos de vegetación de la Sierra de San Pedro Mártir, Baja California. Acta Bot. Mex. 29: 1-30.

Peinado, M., F. Alcaraz, J. L. Aguirre y J. Delgadillo Rodríguez. 1995a. Major plant associations of warm North American Deserts. J. Veg. Sci. 6: 79-94.

Peinado, M., F. Alcaraz y J. Delgadillo Rodríguez. 1995b. Syntaxonomy of some halophilous communities of North and Central America. Phytocoenologia 25: 23-31.

Peinado, M., F. Alcaraz, J. L. Aguirre, J. Delgadillo Rodríguez y J. Álvarez. 1995c. Similarity of zonation within Californian-Baja Californian and Mediterranean salt marshes. Southwest. Nat. 40: 388-405.

Peinado, M., F. Alcaraz, J. L. Aguirre, J. Delgadillo Rodríguez e I. Aguado. 1995d. Shrubland formations and associations in Mediterranean-Desert transitional zones of Northwestern Baja California. Vegetatio 117: 165-179.

Peinado, M., F. Alcaraz, J. L. Aguirre y J. Delgadillo Rodríguez. 1997a. Phytosociological, bioclimatic and biogeographical classification of woody climax communities of western North America. J. Veg. Sci. 8: 505-528.

Peinado, M., F. Alcaraz, J. L. Aguirre y J. M. Martínez-Parras. 1997b. Vegetation formations and associations of the zonobiomes along the North American Pacific coast: from Northern California to Alaska. Plant Ecol. 129: 29-47.

Peinado, M., J. Delgadillo Rodríguez y J. L. Aguirre. 2005. Plant associations of the El Vizcaíno Biosphere Reserve, Baja California Sur, Mexico. Southw. Nat. 50(2): 129149.

Peinado, M., M. Á. Macías, J. Delgadillo Rodríguez y J. L. Aguirre. 2006. Major plant communities of North America's most arid region: the San Felipe Desert, Baja California, Mexico. Plant Bios. 140(3): 280-296.

Peinado, M., J. L. Aguirre, J. Delgadillo Rodríguez y M. Á. Macías. 2007. Zonobiomes, zonoecotones and azonal vegetation along the Pacific coast of North America. Plant Ecol. 191(2): 221-252.

Peinado, M., J. L. Aguirre, J. Delgadillo Rodríguezy M. Á. Macías. 2008. A phytosociological and phytogeographical survey of the coastal vegetation of western North America. Part I: plant communities of Baja California, Mexico. Plant Ecol. 196: 27-60.

Peinado, M., M. Á. Macías, J. L. Aguirre y J. Delgadillo Rodríguez. 2009. Fitogeografía de la costa del Pacífico de Norteamérica. Anales Jard. Bot. Madrid 66(2): 151-194.

Peinado, M., M. Á. Macías, J. L. Aguirre y J. Delgadillo Rodríguez. 2010. Bioclimatevegetation interrelations in Northwestern Mexico. Southw. Nat. 55(3): 311-322. 
Macías Rodríguez et al.: Clasificación bioclimática de la vertiente del Pacífico mexicano

Peinado, M., M. Á. Macías, J. L. Aguirre y J. Delgadillo Rodríguez. 2011. Bioclimates and vegetation along the Pacific basin of Northwestern Mexico. Plant Ecol. 212: 263-281.

Pennington, T. D. y J. Sarukhán. 2005. Árboles tropicales de México. Manual para la identificación de las principales especies. 3a. ed. Universidad Nacional Autónoma de México y Fondo de Cultura Económica. México, D.F., México. 523 pp.

Rivas-Martínez, S. 1997. Syntaxonomical synopsis of the North America natural potential vegetation communities, I. Itinera Geobot. 10: 5-148.

Rivas-Martínez, S., S. Rivas Sáenz y A. Penas. 2011. Worldwide bioclimatic classification system. Global Geobot. 1: 1-634.

Rivas-Martímez, S., D. Sánchez-Mata y M. Costa. 1999. North America boreal and western temperate forest vegetation (Syntaxonomical synopsis of the potential natural plant communities of North America II). Itinera Geobot. 12: 5-316.

Ruiz, J. A., I. J. González, J. Anguiano, I. Vizcaíno, D. Ibarra, J. Alcalá, S. Espinoza y H. E. Flores. 2003. Estadísticas climatológicas básicas para el estado de Jalisco (Período 1961-2000). Libro Técnico Núm. 1. Instituto Nacional de Investigaciones Forestales, Agrícolas y Pecuarias-Centro de Investigación Regional del Pacífico Centro. Tlajomulco de Zuñiga, México. 281 pp.

Ruiz, J. A., G. Medina, J. Grageda, M. M. Silva y G. Díaz. 2005a. Estadísticas climatológicas básicas del estado de Sonora (Período 1961-2003). Libro Técnico Núm. 1. Instituto Nacional de Investigaciones Forestales, Agrícolas y Pecuarias-Centro de Investigación Regional del Noroeste. Ciudad Obregón, México. 170 pp.

Ruiz, J. A., G. Medina, J. Macías, M. M. Silva y G. Díaz. 2005b. Estadísticas climatológicas básicas del estado de Sinaloa (Período 1961-2003). Libro Técnico Núm. 2. Instituto Nacional de Investigaciones Forestales, Agrícolas y Pecuarias-Centro de Investigación Regional del Noroeste. Ciudad Obregón, México. 151 pp.

Ruiz, J. A., G. Díaz, S. D. Guzmán, G. Medina y M. M. Silva. 2006a. Estadísticas climatológicas básicas del estado de Baja California (Período 1961-2003). Libro Técnico Núm. 1. Instituto Nacional de Investigaciones Forestales, Agrícolas y Pecuarias-Centro de Investigación Regional del Noroeste. Ciudad Obregón, México. $165 \mathrm{pp}$.

Ruiz, J. A., G. Medina, R. Meza, G. Díaz y V. Serrano. 2006b. Estadísticas climatológicas básicas del estado de Baja California Sur (Período 1961-2003). Libro Técnico Núm. 2. Instituto Nacional de Investigaciones Forestales, Agrícolas y Pecuarias-Centro de Investigación Regional del Noroeste. Ciudad Obregón, México. 268 pp.

Rzedowski, J. 1978. Vegetación de México. Limusa. México, D.F., México. 432 pp.

Rzedowski, J. y G. Calderón. 2013. Datos para la apreciación de la flora fanerogámica del bosque tropical caducifolio de México. Acta Bot. Mex. 102: 1-23.

Rzedowski, J. y R. McVaugh. 1966. La vegetación de Nueva Galicia. Contr. Univ. Michigan Herb. 9(1): 1-123.

Sánchez-Mejía, Z. M., L. Serrano-Grijalva, P. Peñuelas-Rubio, E. R. Pérez-Ruiz, E. Sequeiros-Ruvalcaba y M. T. García-Calleja. 2007. Composición florística y estructura de la comunidad vegetal del límite del desierto de Sonora y la selva baja caducifolia (Noroeste de México). Rev. Lat. Rec. Nat. 3(1): 74-83. 
Sarukhán, J. 2005. Los tipos de vegetación arbórea de la zona cálido-húmeda de México. In: Pennington, T. D. y J. Sarukhán (eds.). Manual para la identificación de los principales árboles tropicales de México. Instituto Nacional de Investigaciones ForestalesOrganización de las Naciones Unidas para la Agricultura y la Alimentación. México, D.F., México. pp. 3-46.

Serrano, V., G. Díaz, A. López, M. Á. Cano, A. D. Báez y E. R. Garrido. 2006. Estadísticas climatológicas básicas del estado de Chiapas (período 1961-2003). Libro Técnico Núm. 1. Instituto Nacional de Investigaciones Forestales, Agrícolas y PecuariasCentro de Investigación Regional Pacífico Sur. Ocozocoautla de Espinosa, México. $186 \mathrm{pp}$.

Serrano, V., G. Medina, G. Díaz, A. Ruíz y M. Á. Cano. 2007. Estadísticas climatológicas básicas del estado de Guerrero (período 1961-2003). Libro Técnico Núm. 7. Instituto Nacional de Investigaciones Forestales, Agrícolas y Pecuarias-Centro de Investigación Regional Pacífico Sur. Santo Domingo Barrio Bajo, Etla, México. 233 pp.

Serrano, V., M. M. Silva, M. Á. Cano, G. Medina y A. Ruiz. 2005. Estadísticas climatológicas básicas del estado de Oaxaca (período 1961-2003). Libro Técnico Núm. 4. Instituto Nacional de Investigaciones Forestales, Agrícolas y Pecuarias-Centro de Investigación Regional Pacífico Sur. Oaxaca, México. 272 pp.

Shreve, F. 1951. Vegetation of the Sonoran Desert. Carnegie Inst. Wash. 591: 1-192.

Shreve, F. y I. L. Wiggins. 1964. Vegetation and flora of the Sonoran Desert. 2 vols. Stanford University Press. Stanford, USA. 1740 pp.

Takhtajan, A. 1986. Floristic regions of the world. University of California Press. Berkeley, USA. $522 \mathrm{pp}$.

Trejo, I. y R. Dirzo 2002. Floristic diversity of Mexican seasonally dry tropical forests. Biodiv. Conserv. 11: 2063-2084.

Tropicos. 2013. Missouri Botanical Garden. <http://www.tropicos.org> (consultado de agosto de 2010 a septiembre 2011).

Tuhkanen, S. 1980. Climatic parameters and indices in plant geography. Acta Phytogeogr. Suec. 67: 1-110.

Turner, R. M., J. E. Bowers y T. L. Burgess. 1995. Sonoran Desert plants. An ecological atlas. The University of Arizona Press. Tucson, USA. 504 pp.

Tüxen, R. 1956. Die heutige potentielle natürliche Vegetation als Genenstand der Vegetationskartierung. Angew. Pflanzensoz. Stolzenau 13: 5-42.

Van Devender, T. R., A. C. Sanders, R. K. Wilson y S. A. Meyer. 2000. Vegetation, flora, and seasons of the Río Cuchujaqui, a tropical deciduous forest near Alamos, Sonora. In: Robichaux, R. H. y D. A. Yetman (eds.). The tropical deciduous forest of Alamos. Biodiversity of a threatened ecosystem in Mexico. The University of Arizona Press. Tucson, USA. pp. 36-101.

Vizcaíno, I., J. A. Ruiz, J. Anguiano y I. J. González. 2007. Estadísticas climatológicas básicas y clasificación climática del estado de Colima (Periodo 1961-2002). Libro Técnico Núm. 1. Instituto Nacional de Investigaciones Forestales, Agrícolas y Pecuarias-Centro de Investigación Regional del Pacífico Centro. Tecomán, México. $132 \mathrm{pp}$. 
Macías Rodríguez et al.: Clasificación bioclimática de la vertiente del Pacífico mexicano

Walter, H. 1985. Vegetation of the earth and ecological systems of the geobiosphere. 3a. ed. Springer-Verlag. Berlin, Alemania. $318 \mathrm{pp}$.

Westhoff, V. y E. van der Maarel. 1973. The Braun-Blanquet approach. In: Whittaker, R. H. (ed.). Ordination and classification of communities. Dr. W. Junk Publishers. The Hague, Holanda. pp. 617-626.

Wiggins, I. L. 1980. Flora of Baja California. Stanford University Press. Stanford, USA. $1025 \mathrm{pp}$. 